DOI 10.4171/JEMS/290

N. Fusco $\cdot$ A. Pratelli

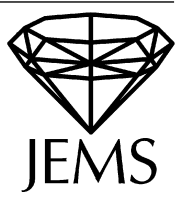

\title{
On a conjecture by Auerbach
}

Received January 21, 2010 and in revised form September 22, 2010

Abstract. In 1938 Herman Auerbach published a paper where he showed a deep connection between the solutions of the Ulam problem of floating bodies and a class of sets studied by Zindler, which are the planar sets whose bisecting chords all have the same length. In the same paper he conjectured that among Zindler sets the one with minimal area, as well as with maximal perimeter, is the so-called "Auerbach triangle". We prove this conjecture.

\section{Contents}

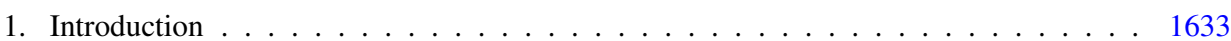

1.1. History of the problem . . . . . . . . . . . . . . . . . . 1633

1.2. Connection with a problem by Santaló . . . . . . . . . . . . . . . . 1635

1.3. Plan of the paper . . . . . . . . . . . . . . . . . . . . . . . 1636

2. Characterization of the Zindler sets . . . . . . . . . . . . . . . . . . . 1638

3. Proof of Theorem $2 \ldots \ldots \ldots \ldots$. . . . . . . . . . . . . . . . 1647

3.1. An $L^{\infty}$ estimate . . . . . . . . . . . . . . . . . . . . . . . . . . . . 1647

3.2. The existence of three "special" angles . . . . . . . . . . . . . . . . . . . . . 1653

4. Proof of Theorem $3 \ldots \ldots$. . . . . . . . . . . . . . . . . . . . . . . 1660

4.1. Preliminary results . . . . . . . . . . . . . . . . 1661

4.2. Step 0 of the proof: no "large" intervals . . . . . . . . . . . . . . . . . . . 1669

4.3. Step 1 of the proof: one "large" interval . . . . . . . . . . . . . . . . . . . 1669

4.4. Step 2 of the proof: two "large" intervals . . . . . . . . . . . . . . . . . 1671

References . . . . . . . . . . . . . . . . . . 1676

\section{Introduction}

\subsection{History of the problem}

In the 1930's, the Polish mathematician S. Ulam proposed the following problem. Let us consider a homogeneous right cylinder of density $1 / 2$, whose section $C$ is a bidimensional convex set. Immerse the cylinder in water in any position such that

(a) the section $C$ is perpendicular to the horizontal plane of water;

(b) the immersed part has half of the total volume.

N. Fusco: Dipartimento di Matematica ed Applicazioni, via Cintia, 80126 Napoli, Italy; e-mail: nicola.fusco@unina.it

A. Pratelli: Dipartimento di Matematica, via Ferrata 1, 27100 Pavia, Italy; e-mail: aldo.pratelli@unipv.it 

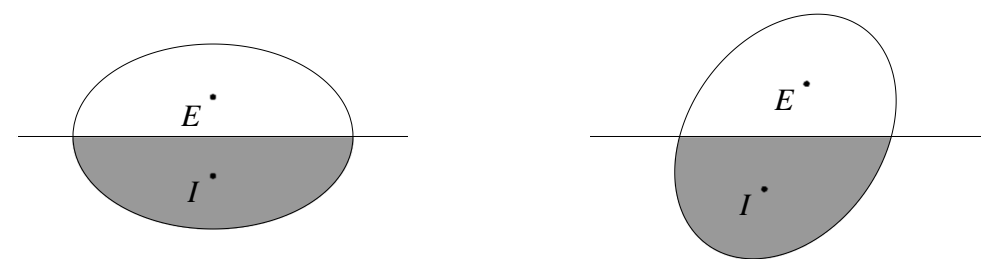

Fig. 1. An equilibrium position (left) and a non-equilibrium position (right).

It is clear that the cylinder is in an equilibrium position if and only if the segment joining the barycenter $I$ of the immersed part and the barycenter $E$ of the external part is vertical (see Figure 1).

Ulam's question was the following. Are there convex sets $C$, besides the disk, such that any position of the cylinder satisfying (a) and (b) is an equilibrium position? We shall say that such a set has the Ulam floating property. For instance, the ellipse of Figure 1 does not have this property, since the right position is not an equilibrium position.

Some years before, the German mathematician K. Zindler considered the following problem: find a planar set, besides the disk, with the property that all the bisecting chords, i.e. the chords dividing the set into two parts of equal area, have the same length. Such a set is now called a Zindler set. A well known example found by Zindler himself is shown in Figure 2.

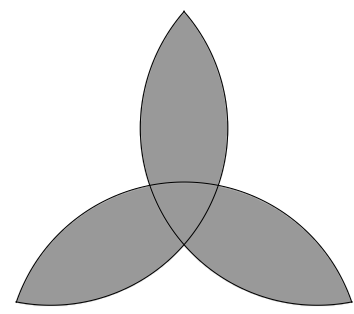

Fig. 2. The "Zindler flower": the three curves are arcs of circle with the same radius whose centers are the vertices of an equilateral triangle.

In 1938 the Polish mathematician H. Auerbach showed the following remarkable connection between the two problems.

Theorem 1 (Auerbach, [1]). A convex planar set has the Ulam floating property if and only if it is a Zindler set. The Ulam floating property is also equivalent to the fact that the locus of all barycenters $I$ and E introduced above is a circle.

In his paper [1], Auerbach shows that the class of convex Zindler sets is large, and provides an explicit analytic description of this class. Moreover, he presents a geometric construction of the set of Figure 3, which we shall refer to as the Auerbach triangle. This set is uniquely characterized, in the class of Zindler sets, by the properties that

- $P P^{\prime}$ and $Q Q^{\prime}$ are bisecting chords of length 2; 
- the curve $P Q$ is a segment;

- $\widehat{P O Q}$ and $\widehat{P^{\prime} O Q^{\prime}}$ are $60^{\circ}$ angles, and the set is invariant under $120^{\circ}$ rotations around $O$.

It is important to mention here that the curve $P^{\prime} Q^{\prime}$ is not an arc of a circle (we will give an analytic definition of this set in Section 2).

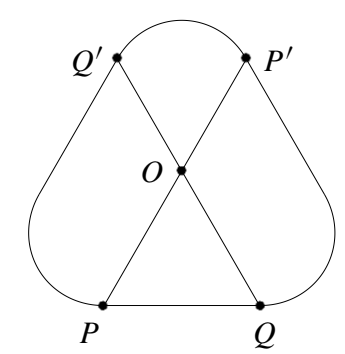

Fig. 3. The "Auerbach triangle".

In his paper, Auerbach raises the question of determining, among all Zindler sets with bisecting chords of given constant length (say 2, so as to compare with the unit disk), those minimizing the area, or maximizing the perimeter. He was not able to answer the question, but he conjectured that at least one of the two extremal sets is his rounded triangle. In his words ([1, p. 138]): Il serait intéressant de déterminer les courbes de périmètre maximé et celles d'aire minimée. Probablement, la courbe triangulaire (Fig. 3) fournit la solution d'au moins un de ces problèmes.

In this paper we prove that the conjecture of Auerbach is correct.

Theorem 2. The Auerbach triangle is the unique set of minimal area in the class of convex Zindler sets with length of bisecting chords equal to 2, up to rotations and translations.

Theorem 3. The Auerbach triangle is the unique set of maximal perimeter in the class of convex Zindler sets with length of bisecting chords equal to 2, up to rotations and translations.

\subsection{Connection with a problem by Santaló}

Various properties of Zindler sets were studied in years past (see for instance [10], [4] and [8]), while the relations between the minimal and maximal length of the bisecting chords and other geometric quantities such as perimeter, diameter, inradius and circumradius have been investigated in more recent times (some results and references can be found in [9], [3], [5] and [7]). For a detailed account on these and on the above mentioned papers on Zindler sets we also refer to Chapters 3 and 4 of [6].

The result proved here is connected to an old question by L. A. Santaló ([2, Problem A26]), who asked whether for any convex set $C$, with area $A(C)$, there always exists a bisecting chord of length at most $2 \sqrt{A(C) / \pi}$ or, equivalently, whether the convex set with minimal bisecting chord of length 2 and smallest area is the unit disk. It is known 
that the answer to Santaló's question is negative, since the area of the Auerbach triangle is less than $\pi$, namely, as shown by Auerbach himself, it is

$$
2 \sqrt{3}\left(\ln (9)-\left(\frac{\ln (3)}{2}\right)^{2}-1\right) \approx 3.102 .
$$

Indeed, as a consequence of formula (1.2) below, every Zindler set other than the disk gives a negative answer to the Santaló problem. However, the question of determining which is the set of minimal area among those with minimal bisecting chord equal to 2 is still open. In this regard, we suppose the following statement is true.

Conjecture. The set minimizing the area in the class of convex sets with bisecting chords of fixed minimal length is a Zindler set. Hence, thanks to Theorem 2, the solution of the Santalo problem would be the Auerbach triangle.

The reason why the above claim is quite reasonable is simple. Consider a convex set $C$ which is not a Zindler set, like in Figure 4, and a bisecting chord not of minimal length, as the horizontal segment depicted in the figure. Then define another set $C_{\varepsilon}$, simply by cutting off an area $\varepsilon$ very close to each end of the bisecting chord (the two shaded portions in the figure). Since the area around both ends is the same, and since the chord was not minimal, it is immediate to realize that the set $C_{\varepsilon}$ has the same minimal bisecting chords as $C$, so that $C$ cannot be a solution of the Santaló problem. Unfortunately, this simple idea does not lead to a formal proof of the conjecture. Indeed, in general (unlike the set in the figure), it may be impossible to cut off the two pieces without strictly decreasing the minimal length of the bisecting chords. This is what may happen if the boundary of $C$ contains a segment, and an endpoint of a minimal chord lies on this segment.

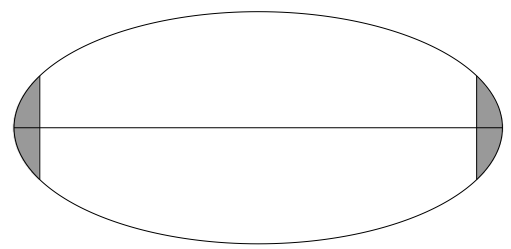

Fig. 4. A convex non-Zindler set $C$ (big set) and a variation $C_{\varepsilon}$ (without the shaded parts).

\subsection{Plan of the paper}

In Section 2 we present an analytic characterization of the convex Zindler sets, quite close to the one given by Auerbach in [1], which shows in particular that those sets are of class $\mathrm{C}^{1,1}$. More precisely, we will give a bijective correspondence between Zindler sets and the class of functions $c: \mathbb{S}^{1} \rightarrow \mathbb{R}$ satisfying, for all $\theta \in \mathbb{S}^{1}$,

$$
\left\{\begin{array}{l}
c(\theta+\pi)=-c(\theta) \\
\int_{\theta}^{\theta+\pi} c(\sigma)(\cos \sigma, \sin \sigma) d \sigma=(0,0) \\
\left|c^{\prime}(\theta)\right| \leq 1+c(\theta)^{2} .
\end{array}\right.
$$


Having this characterization at hand, it is possible to express the area of a Zindler set $C$ by means of the associated function $c$, getting

$$
\operatorname{Area}(C)=\pi-\frac{2}{\pi} \sum_{\substack{n \text { odd } \\ n \geq 3}} \frac{A_{n}^{2}+B_{n}^{2}}{n^{2}-1}=: \pi-\frac{2}{\pi} \mathfrak{F}(c),
$$

$A_{n}$ and $B_{n}$ being the Fourier coefficients of $c$. Hence, denoting by $\hat{c}$ the function corresponding to the Auerbach triangle, Theorem 2 is equivalent to the statement that $\hat{c}$ maximizes $\mathfrak{F}$ among all functions $c$ satisfying (1.1). Since the function $c \equiv 0$ corresponds to the disk, it is evident from (1.2) not only that the disk is not the solution of the Auerbach problem (hence neither, a fortiori, of the Santalo problem), but on the contrary that it maximizes the area among Zindler sets. On the other hand, also the perimeter of the set $C$ can be easily computed in terms of the corresponding function $c$ as

$$
\text { Perimeter }(C)=\int_{0}^{2 \pi} \sqrt{1+c(\theta)^{2}} d \theta .
$$

Hence, it is again clear that the disk, corresponding to $c \equiv 0$, minimizes the perimeter among Zindler sets.

In Section 3 we give the proof of the conjecture about the area, namely Theorem 2. The proof basically consists of two big steps. We start, in Section 3.1, by showing an $L^{\infty}$ estimate for functions $c$ satisfying (1.1), namely that for each such $c$ one has

$$
\|c\|_{L^{\infty}} \leq \tan (\pi / 6) .
$$

In Section 3.2 we will obtain Theorem 2 by showing that indeed $\hat{c}$ maximizes $\mathfrak{F}$ in the larger class of functions $c: \mathbb{S}^{1} \rightarrow \mathbb{R}$ such that

$$
\left\{\begin{array}{l}
c(\theta+\pi)=-c(\theta) \\
|c(\theta)| \leq \tan (\pi / 6) \\
\left|c^{\prime}(\theta)\right| \leq 1+c(\theta)^{2}
\end{array}\right.
$$

The reason why we pass to this new class of functions (which are not necessarily associated to a set) is that this allows more flexibility in defining suitable competitors. We underline that in the proof of the result, and in particular in Section 3.2, we will need to perform extremely careful estimates. This is due to the fact that the difference between the area of the biggest Zindler set (i.e., the area $\pi$ of the ball) and the area of the smallest Zindler set (i.e., the area $\approx 3.102$ of the Auerbach triangle) is just around $1 \%$.

In Section 4 we give the proof of the conjecture about the perimeter, Theorem 3 . The scheme of the proof is completely different from the one of Theorem 2. Indeed, while the proof in the former case hinged on the Fourier coefficients $A_{n}$ and $B_{n}$ of $c$, in this case they do not play any role, simply because there is no reasonable way to express the perimeter in terms of $A_{n}$ and $B_{n}$, as one can easily guess from (1.3). Instead, the proof is based on a careful analysis of the intervals where an optimal function has a costant sign. Roughly speaking, if a function changes sign too often it cannot become big, thus it has 
a small perimeter by (1.3); on the other hand, having the same sign in a too large interval forces again the function to be small, due to the second condition in (1.1). Our argument then consists in taking an optimal function $\bar{c}$ and distinguishing the different cases: all the intervals where $\bar{c}$ has a constant sign are shorter than $\pi / 3$; one of them is longer; two of them are longer. While the first case will be trivial thanks to our estimates of Section 2 , the second one is harder, and the last one is the most involved.

\section{Characterization of the Zindler sets}

The purpose of this section is to show how to associate to any Zindler set a Lipschitz function. Let us start with the relevant definitions.

Definition 2.1. Let $C$ be a bounded, convex subset of $\mathbb{R}^{2}$. For any $\theta \in \mathbb{S}^{1}$, we denote by $M(\theta), R(\theta)$ and $L(\theta)$ the center, the right extreme and the left extreme, respectively, of the unique bisecting chord of $C$ with direction $\theta$. We say that the set $C$ is a Zindler set if all the bisecting chords have the same length.

Throughout the paper we will always consider, with no loss of generality, Zindler sets with bisecting chords of length 2, so as to compare them with the unit disk.

Fix a Zindler set $C$ and notice that, since the bisecting chords of directions $\theta$ and $\theta+\pi$ are the same segment with opposite orientation, one has

$$
M(\theta)=M(\theta+\pi), \quad R(\theta)=L(\theta+\pi), \quad L(\theta)=R(\theta+\pi) .
$$

First, we show that the curve $\theta \mapsto M(\theta)$ is differentiable almost everywhere.

Lemma 2.2. Let $C$ be a Zindler set and let $\theta \in \mathbb{S}^{1}$ be such that $\partial C$ has a unique tangent at both $R(\theta)$ and $L(\theta)$. Then the curve $M$ is differentiable at $\theta$ and

$$
M^{\prime}(\theta)=c(\theta)(\cos \theta, \sin \theta)
$$

for some $c(\theta) \in \mathbb{R}$.

Proof. Assuming $\theta=0$ and $M(0)=(0,0)$ just to get simpler notation and figure, we take a small angle $\varepsilon$ and define $a=a(\varepsilon), b=b(\varepsilon), \alpha=\alpha(\varepsilon)$ and $\beta=\beta(\varepsilon)$ as in Figure 5, that is, in such a way that

$$
R(\varepsilon)=(1+a, b), \quad L(\varepsilon)=(-1+\alpha,-\beta) .
$$

Notice that $a, b, \alpha$ and $\beta$ tend to 0 as $\varepsilon \rightarrow 0$. Since $C$ is a Zindler set, we know that

$$
2=\sqrt{(2+a-\alpha)^{2}+(b+\beta)^{2}},
$$

from which we deduce

$$
a=\alpha+o(|a|+|b|+|\alpha|+|\beta|) \text {. }
$$




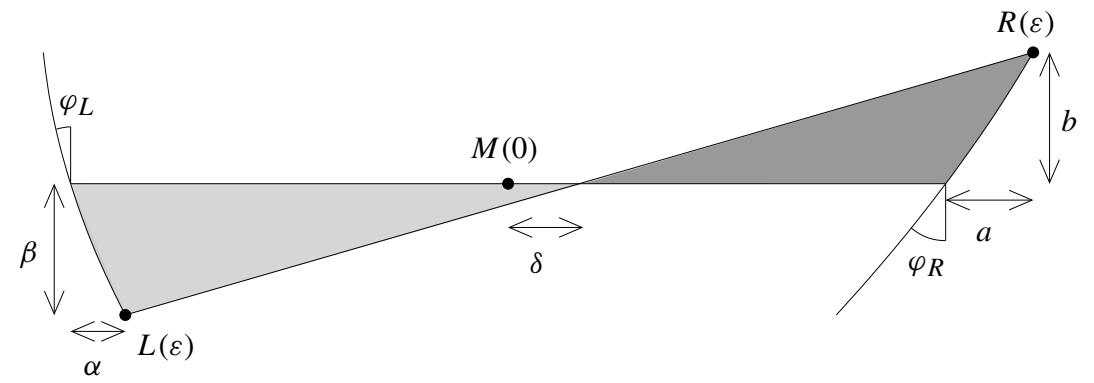

Fig. 5. The situation considered in the proof of Lemma 2.2.

Moreover, from the assumption that $\partial C$ admits a tangent at $L(0)$ and $R(0)$ we know that

$$
a=b \tan \left(\varphi_{R}\right)+o(|b|), \quad \alpha=\beta \tan \left(\varphi_{L}\right)+o(|\beta|)
$$

where, as in Figure 5, $\varphi_{L}$ and $\varphi_{R}$ are the angles between the tangent to $\partial C$ at $L(0)$ and $R(0)$ and the direction $\pi / 2$. By the convexity of $C$ and the fact that $L(0) R(0)$ bisects the set $C$, we also have $-\pi / 2<\varphi_{L}, \varphi_{R}<\pi / 2$. This allows us to rewrite (2.2) as

$$
a=\alpha+o(|b|+|\beta|) .
$$

Define now, again as in the figure, $\delta=\delta(\varepsilon)$ in such a way that $(\delta, 0)$ is the intersection between the chords $L(0) R(0)$ and $L(\varepsilon) R(\varepsilon)$. Then by elementary geometry, we have

$$
\frac{1-\delta+a}{b}=\frac{1+\delta-\alpha}{\beta}
$$

which gives

$$
\beta=b+\delta(\beta+b)+o(|b|+|\beta|) .
$$

Finally, recall that the two chords at the angles 0 and $\varepsilon$ are both bisecting chords. Therefore, the area of the left and right part of $C$ enclosed by the two chords (the light and dark colored regions in the figure) is the same. Writing down this equality, and keeping in mind that $b$ and $\beta$ have by construction the same sign, we obtain

$$
\frac{1}{2}(1+\delta) \beta+o(|\beta|)=\frac{1}{2}(1-\delta) b+o(|b|),
$$

from which we deduce

$$
\beta=b-\delta(\beta+b)+o(|b|+|\beta|) .
$$

Putting together (2.5) and (2.6) one immediately finds

$$
b=\beta+o(|b|+|\beta|) .
$$

From (2.4) and (2.7) we get

$$
\lim _{\varepsilon \rightarrow 0} \frac{a}{b}=\lim _{\varepsilon \rightarrow 0} \frac{\alpha}{\beta},
$$


which in view of (2.3) tells us that $\varphi_{R}=\varphi_{L}=: \varphi$ (by the way, this "geometrical" property of Zindler sets is interesting in itself).

Since, by definition, the chord connecting $L(\varepsilon)$ and $R(\varepsilon)$ has direction $\varepsilon$, we can finally write the values of $a, b, \alpha$ and $\beta$ as

$$
a=\varepsilon \tan \varphi+o(\varepsilon), \quad b=\varepsilon+o(\varepsilon), \quad \alpha=\varepsilon \tan \varphi+o(\varepsilon), \quad \beta=\varepsilon+o(\varepsilon) .
$$

As a consequence, the center $M(\varepsilon)$ of the bisecting chord of direction $\varepsilon$ has coordinates

$$
M(\varepsilon) \equiv(\varepsilon \tan \varphi+o(\varepsilon), o(\varepsilon)) .
$$

This shows that the curve $M$ is differentiable at 0 , and that its velocity is $M^{\prime}(0)=$ $(\tan \varphi, 0)$, hence has the direction of $\theta=0$. Since the choice of $\theta$ was arbitrary, setting $c(\theta):=\tan \varphi$ concludes the proof.

Remark 2.3. The construction above, and in particular formula (2.8) for $a, b, \alpha$ and $\beta$, shows another important property of Zindler sets $C$ : all the bisecting chords divide $C$ into two parts not just with the same area, but also with the same perimeter.

Lemma 2.4. The function $c: \mathbb{S}^{1} \rightarrow \mathbb{R}$ defined by (2.1) is Lipschitz, and for a.e. $\theta \in \mathbb{S}^{1}$,

$$
\left\{\begin{array}{l}
c(\theta+\pi)=-c(\theta) ; \\
\int_{\theta}^{\theta+\pi} c(\sigma)(\cos \sigma, \sin \sigma) d \sigma=(0,0) \\
\left|c^{\prime}(\theta)\right| \leq 1+c(\theta)^{2} .
\end{array}\right.
$$

Proof. Consider the function $\varphi$ introduced in the proof of Lemma 2.2, and recall the relation between $c$ and $\varphi: c(\theta)=\tan (\varphi(\theta))$. We can start showing the first equality in (2.9). Indeed, we immediately get even something more, namely

$$
c(\theta+\pi)=-c(\theta), \quad \varphi(\theta+\pi)=-\varphi(\theta) .
$$

The left equality follows from (2.1) since $M(\theta+\pi)=M(\theta)$ for all $\theta$. As a consequence, since by construction $-\pi / 2<\varphi(\theta)<\pi / 2$, we also obtain the right equality. Notice that one can also get the latter equality by a direct geometric argument based on the construction above and then deduce the former by definition.

Recall now that, by (2.1), for any $\theta, \tilde{\theta} \in \mathbb{S}^{1}$ one has

$$
M(\tilde{\theta})=M(\theta)+\int_{\theta}^{\tilde{\theta}} c(\sigma)(\cos \sigma, \sin \sigma) d \sigma .
$$

Applying this formula with $\tilde{\theta}=\theta+\pi$ and recalling that $M(\theta+\pi)=M(\theta)$, we get the second equality in (2.9). Notice that the equality just proven implies the first one.

Finally, we observe that the tangent line to $\partial C$ at $R(\theta)$ has direction

$$
\tau(\theta)=\theta+\pi / 2-\varphi(\theta),
$$


and by the convexity of $C$ the function $\theta \mapsto \tau(\theta)$ must be increasing. This implies that $\theta-\varphi(\theta)$ is an increasing function of $\theta$, and then for any $\theta \in \mathbb{S}^{1}$ and any $\varepsilon>0$ one has

$$
\varphi(\theta+\varepsilon)-\varphi(\theta)<\varepsilon .
$$

Since this inequality also holds for $\theta+\pi$, keeping in mind (2.10) we get

$$
\varphi(\theta+\varepsilon)-\varphi(\theta)>-\varepsilon,
$$

thus showing that $\varphi$ is 1-Lipschitz. Since by definition $c(\theta)=\tan (\varphi(\theta))$, we see that $c$ is Lipschitz and that the last estimate in (2.9) holds, thus concluding the proof.

Remark 2.5. It is interesting to underline a couple of geometric consequences of Lemmas 2.2 and 2.4. Any Zindler set has a $\mathrm{C}^{1,1}$ boundary and has the property that any bisecting chord makes the same angle with the tangents at the two endpoints. This second property was already observed in proving Lemma 2.2 , while the first one follows from the fact that $\varphi$ is Lipschitz, as established in the proof of Lemma 2.4, and the fact that each point of the boundary of $C$ is the right endpoint of a unique bisecting chord. It is worth observing that what really prevents the boundary of a Zindler set from having a corner is the second of these two properties. Indeed, if there were a "convex" corner at one endpoint of a bisecting chord, there should also be an opposite "concave" corner at the other endpoint of the same chord, contrary to the convexity of $C$.

We can now show the remarkable fact that the three equations in (2.9) characterize all the convex Zindler sets, that is, given any function $c$ satisfying (2.9), there is a convex Zindler set $C$ corresponding to the function $c$.

Proposition 2.6. The map associating to any convex Zindler set $C$ the function $c$ for which (2.1) holds is a one-to-one correspondence between the convex Zindler sets $C$ (up to translations) and the functions $c: \mathbb{S}^{1} \rightarrow \mathbb{R}$ satisfying (2.9).

Proof. The fact that the functions $c$ given by (2.1) satisfy (2.9) has been established in Lemma 2.4 , so we only need to show the opposite implication.

Let then $c$ be any function for which (2.9) holds and define $L, R: \mathbb{S}^{1} \rightarrow \mathbb{R}^{2}$ by

$$
\begin{aligned}
& L(\theta)=\int_{0}^{\theta} c(\sigma)(\cos \sigma, \sin \sigma) d \sigma-(\cos \theta, \sin \theta), \\
& R(\theta)=\int_{0}^{\theta} c(\sigma)(\cos \sigma, \sin \sigma) d \sigma+(\cos \theta, \sin \theta) .
\end{aligned}
$$

By the second property of (2.9) one has $R(\theta+\pi)=L(\theta)$, and $\theta \mapsto R(\theta)$ is a $\mathrm{C}^{1}$ closed curve in $\mathbb{R}^{2}$ with

$$
R^{\prime}(\theta)=c(\theta)(\cos \theta, \sin \theta)+(-\sin \theta, \cos \theta) .
$$

The fact that this curve is the boundary of a convex set $C$ will follow at once by proving that

$$
\theta \mapsto \frac{R^{\prime}(\theta)}{\left|R^{\prime}(\theta)\right|}
$$


is a one-to-one increasing map from $\mathbb{S}^{1}$ to itself, and this, in turn, can be derived, after some straightforward calculations, from the third inequality in (2.9).

To show that $C$ is a Zindler set, it is enough to check that for any $\theta$ the segment $L(\theta) R(\theta)$ is the bisecting chord of direction $\theta$ and length 2. The fact that $L(\theta) R(\theta)$ has length 2 and direction $\theta$ is obvious by (2.11). Moreover, the area of the portion of $C$ above $L(\theta) R(\theta)$ is constant with respect to $\theta$ because its derivative is

$$
\frac{1}{2}\left(R^{\prime}(\theta)+L^{\prime}(\theta)\right) \cdot(-\sin \theta, \cos \theta)=0,
$$

as can be checked by geometrical considerations as in Lemma 2.2. Since by (2.9), $L(\theta+\pi)$ $=R(\theta)$ and $R(\theta+\pi)=L(\theta)$, we deduce that the segments $L(\theta) R(\theta)$ are bisecting.

To conclude the proof we observe that $c$ is exactly the function associated to $C$ by (2.1), and this is in turn true since the center $M(\theta)$ of the bisecting chord of direction $\theta$ is given by

$$
M(\theta)=\frac{L(\theta)+R(\theta)}{2}=\int_{0}^{\theta} c(\sigma)(\cos \sigma, \sin \sigma) d \sigma,
$$

so that $M^{\prime}(\theta)=c(\theta)(\cos \theta, \sin \theta)$, according to $(2.1)$.

Having reduced the problem to the study of a suitable class of Lipschitz functions, it is important to get a useful formula for the perimeter and the area of the set $C$ in terms of the corresponding function $c$. This will be done in the following lemma.

Lemma 2.7. If $C$ is the Zindler set corresponding to the function $c$, its area is given by

$$
\operatorname{Area}(C)=\pi+\int_{0}^{\pi}\left(\int_{0}^{\theta} c(\theta) c(\sigma) \sin (\theta-\sigma) d \sigma\right) d \theta
$$

and its perimeter by

$$
\operatorname{Perimeter}(C)=2 \int_{0}^{\pi} \sqrt{1+c^{2}(\theta)} d \theta .
$$

Proof. We start by recalling that the area of a convex set $C$ in $\mathbb{R}^{2}$, with boundary $\Gamma$, is given by

$$
\operatorname{Area}(C)=\frac{1}{2} \int_{\Gamma}(x d y-y d x)
$$

Since $C$ is a Zindler set, its boundary is the set of the points $R(\theta)$ for $0 \leq \theta \leq 2 \pi$, and from (2.11) we know that

$$
R(\theta)=\int_{0}^{\theta} c(\sigma)(\cos \sigma, \sin \sigma) d \sigma+(\cos \theta, \sin \theta)
$$

so that on $\Gamma$,

$$
(d x, d y) \equiv(c(\theta)(\cos \theta, \sin \theta)+(-\sin \theta, \cos \theta)) d \theta
$$


Thanks to (2.14), we then have

$$
\begin{aligned}
\operatorname{Area}(C)= & \frac{1}{2} \int_{0}^{2 \pi}\left(\left(\int_{0}^{\theta} c(\sigma) \cos \sigma d \sigma+\cos \theta\right)(c(\theta) \sin \theta+\cos \theta)\right. \\
& \left.-\left(\int_{0}^{\theta} c(\sigma) \sin \sigma d \sigma+\sin \theta\right)(c(\theta) \cos \theta-\sin \theta)\right) d \theta \\
= & \pi+\frac{1}{2} \int_{0}^{2 \pi}\left(\int_{0}^{\theta} c(\sigma) c(\theta) \sin (\theta-\sigma)+c(\sigma) \cos (\theta-\sigma) d \sigma\right) d \theta
\end{aligned}
$$

Using the properties of $c$ listed in (2.9), we see that for any $0 \leq \theta \leq \pi$,

$$
\begin{aligned}
\int_{0}^{\pi+\theta} c(\sigma) \cos ((\pi+\theta)-\sigma) d \sigma & =\int_{\pi}^{\pi+\theta} c(\sigma) \cos ((\pi+\theta)-\sigma) d \sigma \\
& =-\int_{0}^{\theta} c(\sigma) \cos (\theta-\sigma) d \sigma
\end{aligned}
$$

hence the last term in (2.15) is 0 , that is,

$$
\int_{0}^{2 \pi}\left(\int_{0}^{\theta} c(\sigma) \cos (\theta-\sigma) d \sigma\right) d \theta=0
$$

Arguing in the very same way, we have

$$
\begin{aligned}
\int_{0}^{\pi+\theta} c(\sigma) c(\pi+\theta) \sin ((\pi+\theta)-\sigma) d \sigma & =\int_{\pi}^{\pi+\theta} c(\sigma) c(\pi+\theta) \sin ((\pi+\theta)-\sigma) d \sigma \\
& =\int_{0}^{\theta} c(\sigma) c(\theta) \sin (\theta-\sigma) d \sigma
\end{aligned}
$$

which gives

$$
\int_{0}^{2 \pi}\left(\int_{0}^{\theta} c(\sigma) c(\theta) \sin (\theta-\sigma) d \sigma\right) d \theta=2 \int_{0}^{\pi}\left(\int_{0}^{\theta} c(\sigma) c(\theta) \sin (\theta-\sigma) d \sigma\right) d \theta .
$$

Inserting (2.17) and (2.16) in (2.15), one gets the formula (2.12).

Concerning (2.13), on the other hand, it is enough to notice that by (2.11) one has

$$
R^{\prime}(\theta)=c(\theta)(\cos \theta, \sin \theta)+(-\sin \theta, \cos \theta), \text { thus }\left|R^{\prime}(\theta)\right|=\sqrt{1+c^{2}(\theta)},
$$

and to recall that $c(\theta+\pi)=-c(\theta)$.

Formula (2.12) suggests considering the Fourier series of $c$ to treat the area functional. This makes sense because the functions satisfying (2.9) are Lipschitz functions. 
Definition 2.8. Let $c: \mathbb{S}^{1} \rightarrow \mathbb{R}$ satisfy (2.9). For any $n \in \mathbb{N}$, define the Fourier coefficients as

$$
A_{n}:=\frac{1}{2} \int_{0}^{2 \pi} c(\theta) \sin (n \theta) d \theta, \quad B_{n}:=\frac{1}{2} \int_{0}^{2 \pi} c(\theta) \cos (n \theta) d \theta .
$$

Notice that (2.9) implies that $A_{n}=B_{n}=0$ for all even $n$ and for $n=1$. Hence, the Fourier expansion of $c$ becomes

$$
c(\theta)=\frac{2}{\pi} \sum_{\substack{n \text { odd } \\ n \geq 3}}\left(A_{n} \sin (n \theta)+B_{n} \cos (n \theta)\right) .
$$

Moreover, for all odd $n \geq 3$,

$$
A_{n}=\int_{0}^{\pi} c(\theta) \sin (n \theta) d \theta, \quad B_{n}=\int_{0}^{\pi} c(\theta) \cos (n \theta) d \theta .
$$

The unusual definition of the Fourier coefficients given above, which explains the constant $2 / \pi$ in (2.18), has the advantage that the two integrals in (2.19) appear without any multiplicative constant. This will simplify a lot the calculations in the next section.

Remark 2.9. From the well known properties of Fourier series, one sees immediately that for any odd $n \geq 3$ there exists an angle $\bar{\varphi}$ such that

$$
\sqrt{A_{n}^{2}+B_{n}^{2}}=\sup _{\varphi \in \mathbb{S}^{1}} \int_{0}^{\pi} c(\theta) \sin (n \theta+\varphi) d \theta=\int_{0}^{\pi} c(\theta) \sin (n \theta+\bar{\varphi}) d \theta .
$$

Notice also that, by rotating $c$ (that is, passing from $c$ to $\tilde{c}(\theta)=c(\theta+\bar{\theta})$ for some $\bar{\theta}$ ), $A_{n}$ and $B_{n}$ may change, but the quantity $\sqrt{A_{n}^{2}+B_{n}^{2}}$ does not change, since the supremum in (2.20) is attained at $\bar{\varphi}+n \bar{\theta}$ instead of $\bar{\varphi}$, but its value remains the same.

We can now give a more expressive formula for the area of $C$ which makes use of the above notation.

Lemma 2.10. Given a Zindler set $C$ corresponding to the function $c$, one has

$$
\operatorname{Area}(C)=\pi-\frac{2}{\pi} \sum_{\substack{n \text { odd } \\ n \geq 3}} \frac{A_{n}^{2}+B_{n}^{2}}{n^{2}-1}
$$

Proof. Fix $0 \leq \theta \leq \pi$, and calculate

$$
\int_{0}^{\theta} c(\sigma) \sin (\theta-\sigma) d \sigma=\frac{2}{\pi} \int_{0}^{\theta} \sum_{\substack{n \text { odd } \\ n \geq 3}}\left(A_{n} \sin (n \sigma)+B_{n} \cos (n \sigma)\right) \sin (\theta-\sigma) d \sigma .
$$


Since

$$
\begin{aligned}
& \int_{0}^{\theta} \sin (\theta-\sigma) \sin (n \sigma) d \sigma=\frac{n \sin \theta-\sin (n \theta)}{n^{2}-1} \\
& \int_{0}^{\theta} \sin (\theta-\sigma) \cos (n \sigma) d \sigma=\frac{\cos \theta-\cos (n \theta)}{n^{2}-1}
\end{aligned}
$$

for any $\theta \in[0, \pi]$ we have

$$
\int_{0}^{\theta} c(\sigma) \sin (\theta-\sigma) d \sigma=\frac{2}{\pi} \sum_{\substack{n \text { odd } \\ n \geq 3}}\left(A_{n} \frac{n \sin \theta-\sin (n \theta)}{n^{2}-1}+B_{n} \frac{\cos \theta-\cos (n \theta)}{n^{2}-1}\right) .
$$

Inserting this equality in (2.12) we obtain

$$
\begin{aligned}
\operatorname{Area}(C) & =\pi+\frac{2}{\pi} \int_{0}^{\pi} c(\theta) \sum_{\substack{n \text { odd } \\
n \geq 3}}\left(A_{n} \frac{n \sin \theta-\sin (n \theta)}{n^{2}-1}+B_{n} \frac{\cos \theta-\cos (n \theta)}{n^{2}-1}\right) d \theta \\
& =\pi+\frac{4}{\pi^{2}} \int_{0}^{\pi} \sum_{\substack{n, m \text { odd } \\
n, m \geq 3}}\left(A_{m} \sin (m \theta)+B_{m} \cos (m \theta)\right) \\
& =\pi-\frac{2}{\pi} \sum_{\substack{n \text { odd } \\
n \geq 3}} \frac{A_{n}^{2}+B_{n}^{2}}{n^{2}-1} .\left(\frac{A_{n}(n \sin \theta-\sin (n \theta))+B_{n}(\cos \theta-\cos (n \theta))}{n^{2}-1}\right) d \theta
\end{aligned}
$$

We can then reformulate our problem: minimizing the area among all Zindler sets is now reduced to the problem of maximizing the sum

$$
\mathfrak{F}(c):=\sum_{\substack{n \text { odd } \\ n \geq 3}} \frac{A_{n}^{2}+B_{n}^{2}}{n^{2}-1}
$$

among all functions $c: \mathbb{S}^{1} \rightarrow \mathbb{R}$ satisfying (2.9).

Remark 2.11. Thanks to the new formulation of our problem, we see that the ball is certainly not the area minimizer. On the contrary, since $\mathfrak{F}(c)=0$ for the null function $c$ (which corresponds to the ball) and $\mathfrak{F}(c)>0$ for any other function $c$, i.e., for any other Zindler set, we can conclude that the ball is the Zindler set with the largest area.

We conclude this section by introducing $\hat{c}$, which in the next section we will prove to be the optimal function. In the arc $[0, \pi / 6]$ the function is defined by setting

$$
\hat{c}(\theta):=\tan \theta \quad \forall 0 \leq \theta \leq \pi / 6,
$$




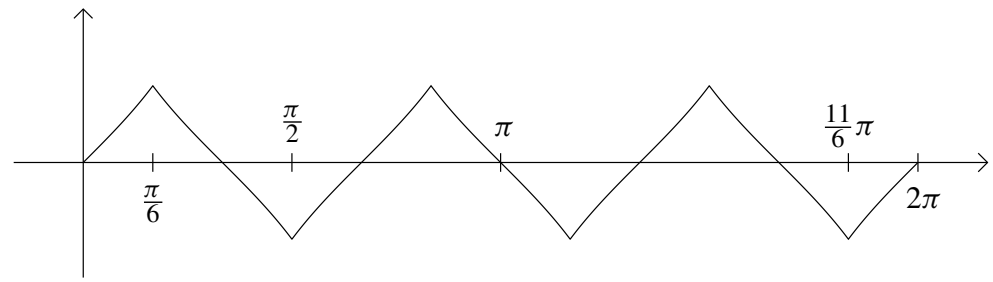

Fig. 6. The optimal function $\hat{c}$.

then it is defined in all $\mathbb{S}^{1}$ by the symmetries shown in Figure 6 . It is immediate to notice that the function thus obtained satisfies (2.9), so it corresponds to a Zindler set. In particular, let us observe that this set is exactly the Auerbach triangle drawn in Figure 7. Indeed, recall that, by the proof of Lemma 2.4 , the local convexity of the boundary of $C$ at the right extreme of the bisecting chord of direction $\theta$ corresponds to the inequality $c^{\prime}(\theta) \leq 1+c^{2}$. For the function $\hat{c}$, one has exactly the equality $\hat{c}^{\prime}=1+\hat{c}^{2}$ in $[-\pi / 6, \pi / 6]$, so the part of the boundary made by the right extremes of the bisecting chords with direction $\theta \in[-\pi / 6, \pi / 6]$ is a segment (the vertical segment on the right of the figure). Moreover, the $120^{\circ}$ symmetry of the set is immediately deduced from the corresponding symmetry of $\hat{c}$. Notice that the set associated to $\hat{c}$ is rotated by $90^{\circ}$ with respect to the one represented in Figure 3. This choice does not affect the minimization problem, but gives a simpler analytic expression to $\hat{c}$.

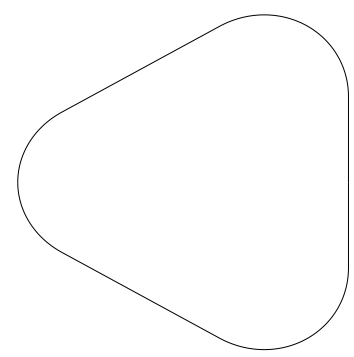

Fig. 7. The optimal set, Auerbach triangle.

Finally, notice that the function $\hat{c}$ is "extremal", in the sense that the inequality $\left|\hat{c}^{\prime}\right| \leq$ $1+\hat{c}^{2}$ is an equality almost everywhere. Moreover, $\hat{c}$ is the "simplest" extremal function satisfying (2.9). More precisely, it is easy to observe that, for any extremal function $c$ satisfying (2.9), the sign of $c^{\prime}$ must change at least three times in the interval $[0, \pi]$ (this can be obtained as an easy consequence of Lemma 3.1 below), and in particular this happens exactly three times if and only if $c=\hat{c}$ up to a rotation. Thanks to this observation, it is somehow not too surprising that $\hat{c}$ has the least area and the maximal perimeter among Zindler sets at once, even though the two extremality properties seem to require very different analyses, as one can suspect from our proofs of Theorems 2 and 3. 


\section{Proof of Theorem 2}

In this section we present the proof of Theorem 2. The main steps of the proof are two. First, we give an $L^{\infty}$ estimate for all functions $c$ satisfying (2.9). Then, via comparison with suitably constructed competitors, we find a general result on the structure of a function which maximizes (2.22). At that point, the proof of the theorem is almost immediate.

\subsection{An $L^{\infty}$ estimate}

In this section we will show a uniform bound for admissible functions $c$ and we will collect some other estimates to be used later on.

In what follows, if $c: \mathbb{S}^{1} \rightarrow \mathbb{R}$, by a rotation of $c$ we mean any function $\tilde{c}: \mathbb{S}^{1} \rightarrow \mathbb{R}$ such that $\tilde{c}(\theta)=c(\theta+\bar{\varphi})$ for some convenient $\bar{\varphi} \in \mathbb{S}^{1}$. Similarly, by the reflection of $c$ we mean the function $\tilde{c}: \mathbb{S}^{1} \rightarrow \mathbb{R}$ such that $\tilde{c}(\theta)=c(\pi-\theta)$ for all $\theta \in \mathbb{S}^{1}$. Notice that in both cases $c$ satisfies (2.9) if and only if $\tilde{c}$ satisfies (2.9).

Proposition 3.1 (The main $L^{\infty}$ estimate). If $c: \mathbb{S}^{1} \rightarrow \mathbb{R}$ satisfies (2.9), then $c \in$ $L^{\infty}\left(\mathbb{S}^{1}\right)$ and the estimate

$$
\|c\|_{L^{\infty}} \leq \tan (\pi / 6)
$$

holds. Moreover, the above inequality is strict, unless $c=\hat{c}$ up to a rotation.

Remark 3.2. Keeping in mind that $\left|c^{\prime}\right| \leq 1+c^{2}$, the $L^{\infty}$ estimate (3.1) implies that all the admissible functions $c$ are uniformly Lipschitz. Correspondingly, all convex Zindler sets, with assigned length of the bisecting chords, are uniformly $\mathrm{C}^{1,1}$.

From this observation, it is immediate to deduce the existence of optimal Zindler sets.

Corollary 3.3 (Existence of extremals). There exist an admissible function minimizing (2.12) and an admissible function maximizing (2.13). Equivalently, there exist a convex Zindler set of minimal area and a convex Zindler set of maximal perimeter.

Proof. As observed in Remark 3.2, the admissible functions are uniformly Lipschitz, so their class is compact under uniform convergence. This implies the conclusion since both area and perimeter are continuous.

The proof of Proposition 3.1 above will be easily obtained from the following technical result.

Lemma 3.4. Let $\bar{\theta} \in(0, \pi / 2)$ and $\tau \in[0, \tan \bar{\theta}]$. Let $c_{\tau, \bar{\theta}}:[0, \pi] \rightarrow \mathbb{R}$ be defined as

$$
c_{\tau, \bar{\theta}}(\theta):= \begin{cases}-\tan (\theta+\arctan \tau) & \text { for } 0 \leq \theta \leq(\bar{\theta}-\arctan \tau) / 2, \\ \tan (\theta-\bar{\theta}) & \text { for }(\bar{\theta}-\arctan \tau) / 2 \leq \theta \leq \pi / 2, \\ \tan (\pi-\bar{\theta}-\theta) & \text { for } \pi / 2 \leq \theta \leq \pi-(\bar{\theta}+\arctan \tau) / 2, \\ \tan (\theta-\pi+\arctan \tau) & \text { for } \pi-(\bar{\theta}+\arctan \tau) / 2 \leq \theta \leq \pi\end{cases}
$$

Then the function

$$
[0, \tan \bar{\theta}] \ni \tau \mapsto \int_{0}^{\pi} c_{\tau, \bar{\theta}}(\theta) \sin \theta d \theta
$$

is strictly increasing. 
Proof. To obtain the assertion, it is enough to fix $0<\tau<\tan \bar{\theta}$ and show that the difference

$$
D:=\int_{0}^{\pi}\left(c_{\tau+\varepsilon, \bar{\theta}}(\theta)-c_{\tau, \bar{\theta}}(\theta)\right) \sin \theta d \theta
$$

is strictly positive for any sufficiently small $\varepsilon>0$.

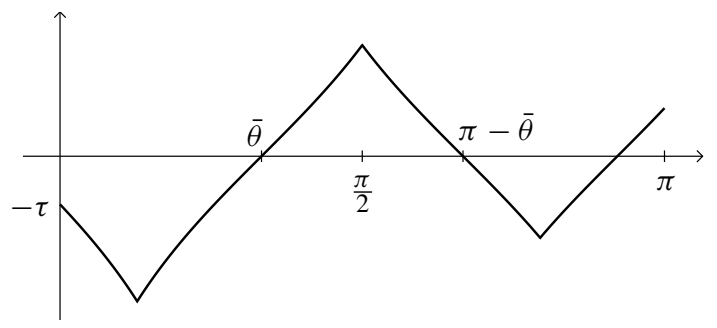

Fig. 8. The graph of $c_{\tau, \bar{\theta}}$ with $\bar{\theta}=\pi / 3$ and $\tau=0.25$.

A first order approximation of $D$ gives

$$
\begin{aligned}
D=\int_{0}^{(\bar{\theta}-\arctan \tau) / 2}\left(c_{\tau+\varepsilon, \bar{\theta}}-c_{\tau, \bar{\theta}}\right)(\theta) \sin \theta & d \theta+\int_{\pi-(\bar{\theta}+\arctan \tau) / 2}^{\pi-\arctan \tau}\left(c_{\tau+\varepsilon, \bar{\theta}}-c_{\tau, \bar{\theta}}\right)(\theta) \sin \theta d \theta \\
& +\int_{\pi-\arctan \tau}^{\pi}\left(c_{\tau+\varepsilon, \bar{\theta}}-c_{\tau, \bar{\theta}}\right)(\theta) \sin \theta d \theta+o(\varepsilon),
\end{aligned}
$$

where the first integral is strictly negative, while the second and third are strictly positive. Thus, to prove that $D$ is strictly positive, it is enough to show that the sum of the first two integrals above is non-negative and since they are both evaluated on intervals of the same length, this will follow if we show that

$$
\left(c_{\tau+\varepsilon, \bar{\theta}}-c_{\tau, \bar{\theta}}\right)(\theta) \sin \theta+\left(c_{\tau+\varepsilon, \bar{\theta}}-c_{\tau, \bar{\theta}}\right)(\pi-\arctan \tau-\theta) \sin (\pi-\arctan \tau-\theta)>0
$$

for all $0 \leq \theta \leq(\bar{\theta}-\arctan \tau) / 2$. An immediate calculation gives that the above inequality can be rewritten, up to the first order, as

$$
-\frac{\varepsilon}{1+\tau^{2}} \frac{1}{\cos ^{2}(\theta+\arctan \tau)} \sin \theta+\frac{\varepsilon}{1+\tau^{2}} \frac{1}{\cos ^{2} \theta} \sin (\pi-\arctan \tau-\theta)>0,
$$

which is in turn equivalent to

$$
\frac{\cos ^{2}(\theta+\arctan \tau)}{\cos ^{2} \theta}>\frac{\sin \theta}{\sin (\theta+\arctan \tau)} \quad \forall 0 \leq \theta \leq(\bar{\theta}-\arctan \tau) / 2 .
$$

Notice that when $\theta$ increases, the left ratio decreases while the right one increases, so to prove this last inequality it is enough to check that it holds when $\theta=(\bar{\theta}-\arctan \tau) / 2=$ : $\bar{\theta} / 2-\alpha$. In conclusion, the statement is reduced to the inequality

$$
\frac{\cos ^{2}(\bar{\theta} / 2+\alpha)}{\cos ^{2}(\bar{\theta} / 2-\alpha)}>\frac{\sin (\bar{\theta} / 2-\alpha)}{\sin (\bar{\theta} / 2+\alpha)} \text {. }
$$

We omit the elementary verification of the inequality above. 
Proof of Proposition 3.1. Let $c$ be a function satisfying (2.9) and such that $\|c\|_{L^{\infty}} \geq$ $\tan (\pi / 6)$. Up to a rotation, we may assume that $c(\pi / 2)=\tan (\pi / 6)$. Moreover, up to a reflection, we can also assume that $\tau:=c(\pi) \geq 0$. Notice also that (2.9) implies that $\tau \leq \tan (\pi / 3)$. Let us observe now that $c \geq c_{\tau, \pi / 3}$ pointwise in $[0, \pi]$. In fact, from the third condition in (2.9) we have $\left|c^{\prime}\right| \leq 1+c^{2}$, so that

$$
\begin{aligned}
& c(\pi / 2)=\tan (\pi / 6) \Rightarrow c(\theta) \geq \tan (2 \pi / 3-\theta) \quad \forall \theta \geq \pi / 2, \\
& c(\pi)=\tau \quad \Rightarrow c(\theta) \geq \tan (\theta-\pi+\arctan \tau) \quad \forall \theta \leq \pi .
\end{aligned}
$$

This proves that $c \geq c_{\tau, \pi / 3}$ in $[\pi / 2, \pi]$, and the proof in $[0, \pi / 2]$ is similar. Since $\sin \theta \geq 0$ in $[0, \pi]$, from the inequality $c \geq c_{\tau, \pi / 3}$ we get

$$
0=\int_{0}^{\pi} c(\theta) \sin \theta d \theta \geq \int_{0}^{\pi} c_{\tau, \pi / 3}(\theta) \sin \theta d \theta \geq \int_{0}^{\pi} c_{0, \pi / 3}(\theta) \sin \theta d \theta=0 .
$$

Notice that the first equality is due to (2.9) and the second inequality is given by Lemma 3.4. The last equality is easy to obtain by observing that $c_{0, \pi / 3}$ (which, by the way, coincides with the function $-\hat{c}$ ) is anti-periodic with period $\pi / 3$, and therefore

$$
\int_{0}^{\pi} c_{0, \pi / 3}(\theta) \sin \theta d \theta=\int_{0}^{\pi / 3} c_{0, \pi / 3}(\theta)(\sin \theta-\sin (\theta+\pi / 3)+\sin (\theta+2 / 3 \pi)) d \theta=0 .
$$

Hence, the two inequalities in (3.2) are indeed equalities and, using again Lemma 3.4, this implies that $c=c_{\tau, \pi / 3}=c_{0, \pi / 3}=-\hat{c}$. This concludes the proof.

Remark 3.5. Notice that all the functions $c_{\tau, \bar{\theta}}$ defined in Lemma 3.4 coincide in $[\bar{\theta}, \pi-\bar{\theta}]$. Hence, arguing as in Proposition 3.1, by Lemma 3.4 we deduce that, for any function $c:[0, \pi] \rightarrow \mathbb{R}$ with

$$
\left|c^{\prime}\right| \leq 1+c^{2}, \quad c(0)=-c(\pi), \quad c(\bar{\theta})=c(\pi-\bar{\theta})=0
$$

we have

$$
\begin{aligned}
\int_{[0, \bar{\theta}] \cup[\pi-\bar{\theta}, \pi]} c(\theta) \sin \theta d \theta & \geq \int_{[0, \bar{\theta}] \cup[\pi-\bar{\theta}, \pi]} c_{\tau, \bar{\theta}}(\theta) \sin \theta d \theta \\
& \geq \int_{[0, \bar{\theta}] \cup[\pi-\bar{\theta}, \pi]} c_{0, \bar{\theta}}(\theta) \sin \theta d \theta
\end{aligned}
$$

where $\tau=|c(\pi)|$.

We now show another consequence of Lemma 3.4, which will be used in Section 4.

Lemma 3.6 (An $L^{\infty}$ estimate in large intervals). Let $0 \leq \bar{\theta}<\pi / 3$, and let $c:[0, \pi] \rightarrow \mathbb{R}$ be a Lipschitz function such that

$$
\begin{gathered}
\left|c^{\prime}\right| \leq 1+c^{2}, \quad \int_{0}^{\pi} c(\theta) \sin \theta d \theta \leq 0, \quad c(0)=-c(\pi), \\
c(\bar{\theta})=c(\pi-\bar{\theta})=0, \quad c \geq 0 \quad \text { in } I,
\end{gathered}
$$

where $I=[\bar{\theta}, \pi-\bar{\theta}]$. Then

$$
\|c\|_{L^{\infty}(I)}<\tan (\bar{\theta} / 2) .
$$


Proof. For contradiction, assume the existence of $\hat{\theta} \in(\bar{\theta}, \pi-\bar{\theta})$ such that $c(\hat{\theta})=$ $\tan (\bar{\theta} / 2)$. Observe that $\hat{\theta} \in[3 \bar{\theta} / 2, \pi-3 \bar{\theta} / 2]$, and that the pointwise inequality

$$
c(\theta) \geq \tilde{c}(\theta):=\chi_{J}(\theta) \tan (\operatorname{dist}(\theta, \partial J))
$$

holds, where $J=(\hat{\theta}-\bar{\theta} / 2, \hat{\theta}+\bar{\theta} / 2)$. We now claim that

$$
\int_{0}^{\pi} c(\theta) \sin \theta d \theta \geq \int_{0}^{\pi} c^{*}(\theta) \sin \theta d \theta,
$$

where $c^{*}:[0, \pi] \rightarrow \mathbb{R}$ is defined by

$$
c^{*}(\theta):= \begin{cases}-\tan \theta & \text { for } 0 \leq \theta \leq \bar{\theta} / 2 \\ \tan (\theta-\bar{\theta}) & \text { for } \bar{\theta} / 2 \leq \theta \leq 3 \bar{\theta} / 2, \\ 0 & \text { for } 3 \bar{\theta} / 2 \leq \theta \leq \pi / 2\end{cases}
$$

and by the relation $c^{*}(\theta)=c^{*}(\pi-\theta)$. The functions $\tilde{c}$ and $c^{*}$ are shown in Figure 9. In
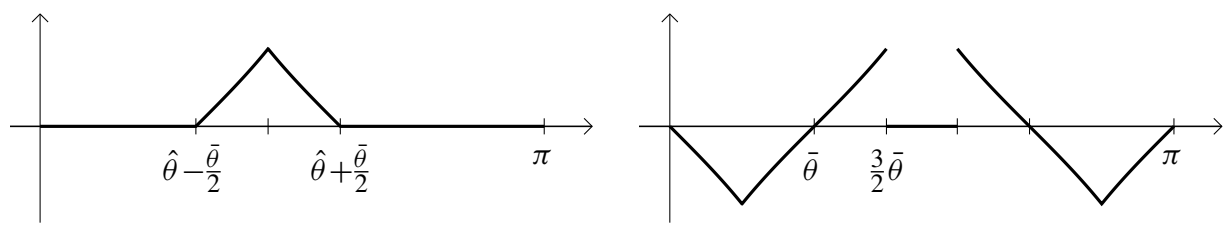

Fig. 9. The functions $\tilde{c}$ (left) and $c^{*}$ (right).

fact, assuming for simplicity that $\hat{\theta} \leq \pi / 2$-which is true up to a reflection-one has

$$
\begin{aligned}
\int_{\bar{\theta}}^{\pi-\bar{\theta}} c(\theta) \sin \theta d \theta & \geq \int_{\bar{\theta}}^{\pi-\bar{\theta}} \tilde{c}(\theta) \sin \theta d \theta \geq 2 \int_{\hat{\theta}-\bar{\theta} / 2}^{\hat{\theta}} \tilde{c}(\theta) \sin \theta d \theta \\
& \geq 2 \int_{\bar{\theta}}^{3 \bar{\theta} / 2} c^{*}(\theta) \sin \theta d \theta=\int_{\bar{\theta}}^{\pi-\bar{\theta}} c^{*}(\theta) \sin \theta d \theta,
\end{aligned}
$$

where the first inequality is due to the pointwise estimate $c \geq \tilde{c}$. Hence, (3.4) follows directly by Remark 3.5 . The proof will thus be completed once we show that

$$
\int_{0}^{\pi} c^{*}(\theta) \sin \theta d \theta>0
$$

To this end we first notice that, for any $0<\lambda<1$, the function

$$
\varphi \mapsto \frac{\sin (\lambda \varphi)}{\sin \varphi}
$$

is strictly increasing in $[0, \pi / 2]$. Therefore, as $\bar{\theta}<\pi / 3$, one has

$$
\sin \left(\frac{3 \bar{\theta}}{\pi} \varphi\right)<\alpha \sin \varphi \quad \forall \varphi \in(0, \pi / 3), \quad \sin \left(\frac{3 \bar{\theta}}{\pi} \varphi\right)>\alpha \sin \varphi \quad \forall \varphi \in(\pi / 3, \pi / 2),
$$


where $\alpha=\sin (\bar{\theta}) / \sin (\pi / 3)$. We now obtain (3.5), thus concluding the proof, since

$$
\begin{gathered}
\int_{0}^{\pi} c^{*}(\theta) \sin \theta d \theta=2 \int_{0}^{3 \bar{\theta} / 2} c^{*}(\theta) \sin \theta d \theta=\frac{6 \bar{\theta}}{\pi} \int_{0}^{\pi / 2} c^{*}\left(\frac{3 \bar{\theta}}{\pi} \varphi\right) \sin \left(\frac{3 \bar{\theta}}{\pi} \varphi\right) d \varphi \\
>\frac{6 \bar{\theta}}{\pi} \alpha \int_{0}^{\pi / 2} c^{*}\left(\frac{3 \bar{\theta}}{\pi} \varphi\right) \sin \varphi d \varphi=\frac{3 \bar{\theta}}{\pi} \alpha \int_{0}^{\pi} c^{*}\left(\frac{3 \bar{\theta}}{\pi} \min (\varphi, \pi-\varphi)\right) \sin \varphi d \varphi=0,
\end{gathered}
$$

where the last equality holds since

$$
\varphi \mapsto c^{*}\left(\frac{3 \bar{\theta}}{\pi} \min (\varphi, \pi-\varphi)\right)
$$

is anti-periodic with period $\pi / 3$.

Now, our task is to show that the optimal function for the area functional provided by Corollary 3.3 coincides with our candidate $\hat{c}$. To this end, the standard strategy would be to argue by contradiction, assuming that the maximum of $\mathfrak{F}$ is attained at some function $\bar{c} \neq \hat{c}$ and finding a suitable competitor of $\bar{c}$ for which $\mathfrak{F}$ is strictly larger. However, in our case this strategy presents a serious difficulty due to the fact that condition (2.9) is not very flexible. Indeed, it is quite hard to build a competitor to an admissible function by making a small variation in such a way as to keep the second constraint in (2.9). To overcome this difficulty, we will show that $\hat{c}$ maximizes (2.22) in a larger and less rigid class of functions. This will be done by using the full strength of the $L^{\infty}$ estimate (3.1).

Definition 3.7. A function $c: \mathbb{S}^{1} \rightarrow \mathbb{R}$ such that $c(\theta+\pi)=-c(\theta)$ for all $\theta \in \mathbb{R}$ will be called weakly admissible if

$$
\left|c^{\prime}(\theta)\right| \leq 1+c^{2}(\theta) \quad \text { and } \quad|c(\theta)| \leq \tan (\pi / 6) \quad \forall 0 \leq \theta \leq 2 \pi .
$$

For any weakly admissible function $c$, the Fourier coefficients are defined as in (2.19).

Thanks to Proposition 3.1, it is obvious that any admissible function, that is, any function satisfying (2.9), is weakly admissible, but it is also clear that the class of weakly admissible functions is wider and more stable. Notice also that to a weakly admissible function which is not admissible there corresponds no Zindler set. Moreover, since also weakly admissible functions are uniformly Lipschitz by definition, as in Corollary 3.3 we have the existence of a weakly admissible function maximizing $\mathfrak{F}$. As anticipated in the Introduction, Theorem 2 will be obtained as a particular case of the following more general result.

Theorem 4. The problem of maximizing $\mathfrak{F}$ among weakly admissible functions admits $\hat{c}$ as a unique solution, up to a rotation.

It is important to emphasize that if $c$ is a weakly admissible function and $A_{n}, B_{n}$ are its Fourier coefficients, there is no reason why $A_{1}$ and $B_{1}$ should be 0 (while of course $A_{n}$ and $B_{n}$ are 0 for all even $n$ ). Hence, the Fourier expansion (2.18) does not hold in general for a weakly admissible function. Nevertheless, notice that in Theorem 4 we consider 
the problem of maximizing $\mathfrak{F}$ as defined by the series (2.22), which adds up the terms with odd $n \geq 3$. In other words, a weakly admissible function may well have non-null coefficients $A_{1}, B_{1}$, but these coefficients do not affect the value of $\mathfrak{F}$.

We conclude this section by collecting a couple of simple but useful estimates to be used later.

Lemma 3.8 (Some estimates). For any weakly admissible function c, and for any odd $n \geq 3$,

$$
\sqrt{A_{n}^{2}+B_{n}^{2}} \leq \frac{8}{3 n} \text {. }
$$

Moreover, if $c$ realizes the maximum of (2.22), then also

$$
\sqrt{A_{3}^{2}+B_{3}^{2}} \geq \alpha>0.598
$$

where $\alpha$ is the constant defined in (3.8) below.

Proof. Let us start with (3.6). Keeping in mind that

$$
\left|c^{\prime}(\theta)\right| \leq 1+c^{2}(\theta) \leq 1+\tan ^{2}(\pi / 6)=4 / 3,
$$

and denoting, as in $(2.20)$, by $\bar{\varphi}=\bar{\varphi}(c, n)$ the angle such that

$$
\sqrt{A_{n}^{2}+B_{n}^{2}}=\int_{0}^{\pi} c(\theta) \sin (n \theta+\bar{\varphi}) d \theta
$$

it is immediate to calculate

$$
\begin{aligned}
\sqrt{A_{n}^{2}+B_{n}^{2}} & =\int_{0}^{\pi} c(\theta) \sin (n \theta+\bar{\varphi}) d \theta=\int_{0}^{\pi} c^{\prime}(\theta) \frac{\cos (n \theta+\bar{\varphi})}{n} d \theta \\
& \leq \frac{4}{3 n} \int_{0}^{\pi}|\cos (n \theta+\bar{\varphi})| d \theta=\frac{8}{3 n}
\end{aligned}
$$

Hence (3.6) is proved. To get the lower estimate (3.7), it is enough to compare an optimal function $c$ with our function $\hat{c}$, whose Fourier coefficients we denote by $\widehat{A}_{n}, \widehat{B}_{n}$. Indeed, since $c$ is optimal we have $\mathfrak{F}(c) \geq \mathfrak{F}(\hat{c})$ and hence, recalling (3.6),

$$
\begin{aligned}
\frac{A_{3}^{2}+B_{3}^{2}}{8} & =\sum_{\substack{n \text { odd } \\
n \geq 3}} \frac{A_{n}^{2}+B_{n}^{2}}{n^{2}-1}-\sum_{\substack{n \text { odd } \\
n \geq 5}} \frac{A_{n}^{2}+B_{n}^{2}}{n^{2}-1} \geq \sum_{\substack{n \text { odd } \\
n \geq 3}} \frac{\widehat{A}_{n}^{2}+\widehat{B}_{n}^{2}}{n^{2}-1}-\sum_{\substack{n \text { odd } \\
n \geq 5}} \frac{64}{9 n^{2}\left(n^{2}-1\right)} \\
& \geq \frac{\widehat{A}_{3}^{2}}{8}-\sum_{\substack{n \text { odd } \\
n \geq 5}} \frac{64}{9 n^{2}\left(n^{2}-1\right)} .
\end{aligned}
$$

Let us then define

$$
\alpha:=\left(\widehat{A}_{3}^{2}-\sum_{\substack{n \text { odd } \\ n \geq 5}} \frac{512}{9 n^{2}\left(n^{2}-1\right)}\right)^{1 / 2},
$$


so that (3.7) will follow once one checks that $\alpha>0.598$, which is a very boring but simple task. Indeed, first of all an elementary exercise yields

$$
\widehat{A_{3}}=6 \int_{0}^{\pi / 6} \tan (\theta) \sin (3 \theta) d \theta=4-6 \ln (\sqrt{3})>0.704 .
$$

Hence, to obtain the desired inequality, one has to write

$$
\sum_{\substack{n \text { odd } \\ n \geq 5}} \frac{512}{9 n^{2}\left(n^{2}-1\right)}=\sum_{\substack{n \text { odd } \\ n=5}}^{15} \frac{512}{9 n^{2}\left(n^{2}-1\right)}+\sum_{\substack{n \text { odd } \\ n \geq 17}} \frac{512}{9 n^{2}\left(n^{2}-1\right)}
$$

and to estimate the last sum from above by

$$
\begin{aligned}
\sum_{\substack{n \text { odd } \\
n \geq 17}} \frac{1}{n^{2}\left(n^{2}-1\right)} & \leq \frac{17^{2}}{17^{2}-1} \sum_{\substack{n \text { odd } \\
n \geq 17}} \frac{1}{n^{4}} \leq \frac{17^{2}}{2\left(17^{2}-1\right)} \int_{15}^{+\infty} \frac{1}{x^{4}} d x \\
& =\frac{17^{2}}{6\left(17^{2}-1\right) 15^{3}} .
\end{aligned}
$$

\subsection{The existence of three "special" angles}

This section is entirely devoted to proving Lemma 3.9 below, which will immediately imply Theorem 4 and then Theorem 2. Up to a rotation we may assume that $A_{3}>0$ and $B_{3}=0$. We then have the following result.

Lemma 3.9 (Three "special" angles). Let c be a weakly admissible function maximizing $\mathfrak{F}$, and assume that $A_{3}>0$ and $B_{3}=0$. Then there exist three angles $\theta_{1} \in[0, \pi / 3]$, $\theta_{2} \in[\pi / 3,2 \pi / 3]$ and $\theta_{3} \in[2 \pi / 3, \pi]$ such that

$$
c^{\prime}(\theta)= \begin{cases}1+c^{2}(\theta) & \text { in }\left[0, \theta_{1}\right], \\ -\left(1+c^{2}(\theta)\right) & \text { in }\left[\theta_{1}, \theta_{2}\right], \\ 1+c^{2}(\theta) & \text { in }\left[\theta_{2}, \theta_{3}\right], \\ -\left(1+c^{2}(\theta)\right) & \text { in }\left[\theta_{3}, \pi\right] .\end{cases}
$$

Proof. We will divide the proof into three steps.

Step I. Behavior of c near $\pi / 6$.

Let us define $3 \hat{\theta}=\arcsin (0.55) \approx 33^{\circ}$. In this first step we will show the existence of an angle $\theta_{1} \in[\hat{\theta}, \pi / 3-\hat{\theta}]$ such that

$$
c^{\prime}(\theta)= \begin{cases}1+c^{2}(\theta) & \forall \hat{\theta}<\theta<\theta_{1} \\ -1-c^{2}(\theta) & \forall \theta_{1}<\theta<\pi / 3-\hat{\theta}\end{cases}
$$


Assume that this is not true. Then there exists $\bar{\theta} \in[\hat{\theta}, \pi / 3-\hat{\theta}]$ such that the right derivative of $c$ at $\bar{\theta}$ is not $-1-c^{2}(\bar{\theta})$ (that is, the right derivative either does not exist or is strictly larger than $-1-c^{2}(\bar{\theta})$ ), and the left derivative of $c$ at $\bar{\theta}$ is not $1+c^{2}(\bar{\theta})$ (that is, again, the left derivative either does not exist or is strictly smaller than $\left.1+c^{2}(\theta)\right)$. It is then possible, for arbitrarily small $\varepsilon>0$, to find a function $\tilde{c} \geq c$ on $[0, \pi]$ such that the set $\{\tilde{c}>c\}$ is an arbitrarily small interval around $\bar{\theta}$ and

$$
\int_{0}^{\pi}(\tilde{c}(\theta)-c(\theta)) d \theta=\varepsilon, \quad\left|\tilde{c}^{\prime}\right| \leq 1+\tilde{c}^{2} .
$$

The function $\tilde{c}$ can be simply defined by fixing a very small positive $\delta=\delta(\varepsilon)$ and then setting, for $\theta \in[\hat{\theta}, \pi / 3-\hat{\theta}]$,

$$
\gamma(\theta):=\left\{\begin{array}{ll}
\tan (\theta-\bar{\theta}+\arctan (c(\bar{\theta})+\delta)) & \text { for } \theta \leq \bar{\theta}, \\
\tan (\bar{\theta}-\theta+\arctan (c(\bar{\theta})+\delta)) & \text { for } \theta \geq \bar{\theta},
\end{array} \quad \tilde{c}:=c \vee \gamma .\right.
$$

We extend $\tilde{c}$ to $[0, \pi] \backslash[\hat{\theta}, \pi / 3-\hat{\theta}]$ as $\tilde{c}(\theta)=c(\theta)$, then to $[\pi, 2 \pi]$ by setting $\tilde{c}(\theta+\pi)=$ $-\tilde{c}(\theta)$ for $\theta \in[0, \pi]$. The functions $c$ and $\tilde{c}$ are shown in Figure 10 (the smaller function is $c$, the larger $\tilde{c}$, and the area of the dark region is $\varepsilon$ ). Notice that, for $\delta$ small, $\tilde{c}$ is a weakly admissible function. To see this, observe that the conditions $\tilde{c}(\theta+\pi)=-\tilde{c}(\theta)$ and the inequality $\left|\tilde{c}^{\prime}(\theta)\right| \leq 1+\tilde{c}^{2}(\theta)$ are satisfied by construction, so we have only to verify the $L^{\infty}$ estimate $|\tilde{c}(\theta)| \leq \tan (\pi / 6)$. Since we may assume $c(\bar{\theta})<\tan (\pi / 6)$ (otherwise by Proposition 3.1 we would have $c=\hat{c}$, thus concluding the proof), to get the $L^{\infty}$ estimate for $\tilde{c}$ it is enough to take $\delta=\|\tilde{c}-c\|_{L^{\infty}}$ sufficiently small. We claim that

$$
\mathfrak{F}(\tilde{c})>\mathfrak{F}(c),
$$

thus getting a contradiction to the optimality of $c$.

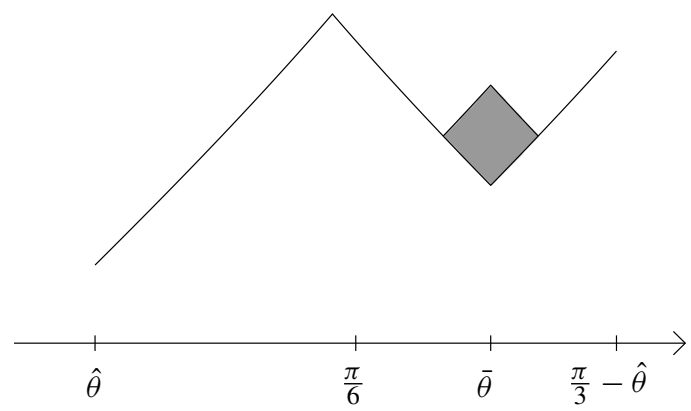

Fig. 10. The functions $c$ and $\tilde{c}$ in Step I: the area of the dark region is $\varepsilon$.

To show (3.11) we start by noticing that, denoting by $\widetilde{A}_{n}, \widetilde{B}_{n}$ the Fourier coefficients of $\tilde{c}$ defined as in (2.19), one has

$$
\widetilde{A}_{3}=A_{3}+\varepsilon \sin (3 \bar{\theta})+o(\varepsilon), \quad \widetilde{B}_{3}=O(\varepsilon) .
$$


Since $A_{3}>0$ and $B_{3}=0$ by assumption, from (3.12) we deduce that the first term in the sum (2.22) defining $\mathfrak{F}(\tilde{c})$ is greater than the first one in $\mathfrak{F}(c)$. Let us now show that the remaining terms are not much smaller than the corresponding ones in $\mathfrak{F}(c)$. Indeed, recalling (2.20) and choosing $\varphi_{n} \in \mathbb{S}^{1}$ such that

$$
\sqrt{A_{n}^{2}+B_{n}^{2}}=\int_{0}^{\pi} c(\theta) \sin \left(n \theta+\varphi_{n}\right) d \theta,
$$

we can estimate $\sqrt{\widetilde{A}_{n}^{2}+\widetilde{B}_{n}^{2}}$ from below as follows:

$$
\begin{aligned}
\sqrt{\widetilde{A}_{n}^{2}+\widetilde{B}_{n}^{2}} & =\sup _{\varphi \in \mathbb{S}^{1}} \int_{0}^{\pi} \tilde{c}(\theta) \sin (n \theta+\varphi) d \theta \geq \int_{0}^{\pi} \tilde{c}(\theta) \sin \left(n \theta+\varphi_{n}\right) d \theta \\
& =\int_{0}^{\pi} c(\theta) \sin \left(n \theta+\varphi_{n}\right) d \theta+\int_{0}^{\pi}(\tilde{c}(\theta)-c(\theta)) \sin \left(n \theta+\varphi_{n}\right) d \theta \\
& =\sqrt{A_{n}^{2}+B_{n}^{2}}+\int_{0}^{\pi}(\tilde{c}(\theta)-c(\theta)) \sin \left(n \theta+\varphi_{n}\right) d \theta \\
& \geq \sqrt{A_{n}^{2}+B_{n}^{2}}-\int_{0}^{\pi}(\tilde{c}(\theta)-c(\theta)) d \theta=\sqrt{A_{n}^{2}+B_{n}^{2}}-\varepsilon .
\end{aligned}
$$

Thus, putting together (3.12) and (3.14), and recalling the estimates (3.6) and (3.7) proved in Lemma 3.8, we get

$$
\begin{aligned}
\mathfrak{F}(\tilde{c})-\mathfrak{F}(c) & =\sum_{\substack{n \text { odd } \\
n \geq 3}} \frac{\widetilde{A}_{n}^{2}+\widetilde{B}_{n}^{2}-A_{n}^{2}-B_{n}^{2}}{n^{2}-1} \\
& \geq \frac{A_{3} \sin (3 \bar{\theta})}{4} \varepsilon+\sum_{\substack{n \text { odd } \\
n \geq 5}} \frac{\widetilde{A}_{n}^{2}+\widetilde{B}_{n}^{2}-A_{n}^{2}-B_{n}^{2}}{n^{2}-1}+o(\varepsilon) \\
& \geq \frac{A_{3} \sin (3 \bar{\theta})}{4} \varepsilon-2 \varepsilon \sum_{\substack{n \text { odd } \\
n \geq 5}} \frac{\sqrt{A_{n}^{2}+B_{n}^{2}}}{n^{2}-1}+o(\varepsilon) \\
& \geq\left(\frac{\alpha \sin (3 \bar{\theta})}{4}-\frac{16}{3} \sum_{\substack{n \text { odd } \\
n \geq 5}} \frac{1}{n\left(n^{2}-1\right)}\right) \varepsilon+o(\varepsilon)
\end{aligned}
$$

where $\alpha$ is the constant defined in (3.8). Recall that, to conclude this step, we need to obtain (3.10), which will follow by contradiction once we reach (3.11). And in turn, since we can choose an arbitrarily small $\varepsilon>0$, we are reduced to checking that the quantity in brackets in the last term of the estimate above is positive. In fact, estimating the sum via a finite sum plus an integral exactly as in the proof of Lemma 3.8, and using the estimate (3.7), it is again boring but elementary to reduce the desired inequality to the inequality $\sin (3 \bar{\theta})>0.542$. Finally, this last inequality holds true since by construction $\sin (3 \bar{\theta}) \geq \sin (3 \hat{\theta})=0.55$. 
Step II. Behavior of c near 0.

Assume now that $c^{\prime}=1+c^{2}$ on $[\hat{\theta}, \pi / 6]$. We claim that in this case one has

$$
c^{\prime}(\theta)=1+c^{2}(\theta) \quad \forall-\hat{\theta} \leq \theta \leq \hat{\theta} .
$$

Indeed, if this is not true, let $\theta_{-}$be the largest angle in $[-\hat{\theta}, \hat{\theta}]$ where the derivative is not $1+c^{2}$, that is,

$$
\theta_{-}=\inf \left\{\eta<\hat{\theta}: c^{\prime}(\theta)=1+c^{2}(\theta) \forall \eta \leq \theta \leq \hat{\theta}\right\}
$$

We are again going to find a competitor, namely a weakly admissible function $\tilde{c}$ such that $\mathfrak{F}(\tilde{c})>\mathfrak{F}(c)$. To this end, fix a small $\varepsilon>0$ and define $\tilde{c}:[-\pi / 2, \pi / 2] \rightarrow \mathbb{R}$ by

$$
\tilde{c}(\theta)= \begin{cases}c(\theta) & \text { for }-\pi / 2 \leq x \leq-\hat{\theta} \\ c(\theta) \vee \tan \left(\theta-\theta_{-}+\arctan \left(c\left(\theta_{-}\right)\right)+\varepsilon\right) & \text { for }-\hat{\theta} \leq x \leq \pi / 6-\varepsilon / 2 \\ \tan \left(-\theta-\theta_{-}+\arctan \left(c\left(\theta_{-}\right)\right)+\pi / 3\right) & \text { for } \pi / 6-\varepsilon / 2 \leq x \leq \pi / 6 \\ c(\theta) & \text { for } \pi / 6 \leq x \leq \pi / 2\end{cases}
$$

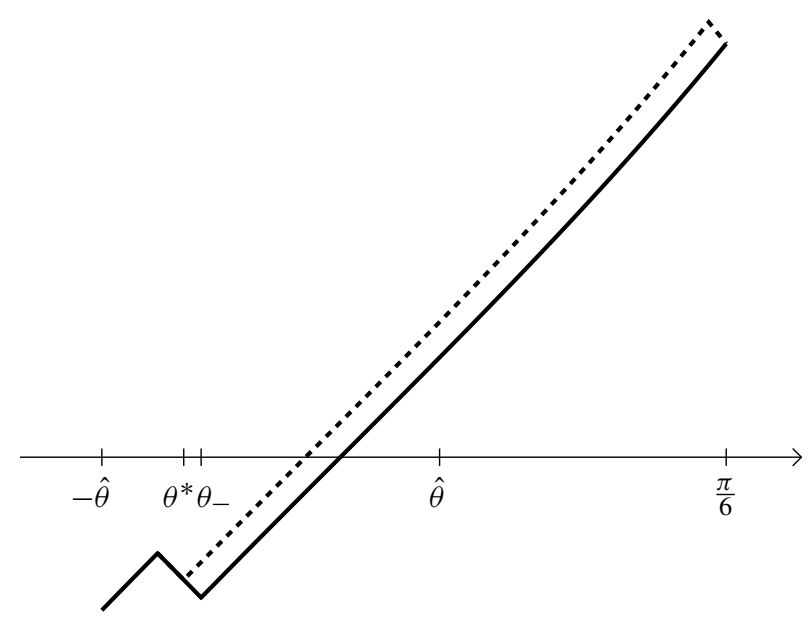

Fig. 11. The functions $c$ (solid) and $\tilde{c}$ (dashed) in Step II.

Notice that

$$
\tilde{c}(\theta)=c(\theta+\varepsilon), \quad \forall \theta_{-} \leq \theta \leq \pi / 6-\varepsilon .
$$

Thus, recalling that $c^{\prime}=1+c^{2}$ and using the $L^{\infty}$ estimate (3.1), we immediately deduce

$$
\varepsilon \leq \tilde{c}(\theta)-c(\theta) \leq 4 \varepsilon / 3 \quad \forall \theta_{-} \leq \theta \leq \pi / 6-\varepsilon .
$$

By construction, $\tilde{c}(\theta)=c(\theta)$ for $\theta \geq \pi / 6$ and, by the definition of $\theta_{-}, \tilde{c}(\theta)=c(\theta)$ in $\left[-\pi / 2, \theta^{*}\right]$ for some $\theta^{*}<\theta_{-}$such that $\theta_{-}-\theta^{*} \rightarrow 0$ as $\varepsilon \rightarrow 0$. Moreover, as in (3.17) we have $0 \leq \tilde{c}(\theta)-c(\theta) \leq 4 \varepsilon / 3$ for $\theta \in\left(\theta^{*}, \theta_{-}\right)$. As in Step I, we extend $\tilde{c}$ to the whole $\mathbb{S}^{1}$ by defining $c(\theta+\pi)=-c(\theta)$, and exactly as in Step I we find that $\tilde{c}$ 
is a weakly admissible function if $\varepsilon \ll 1$. Figure 11 shows the functions $c$ and $\tilde{c}$ in the interval $[-\hat{\theta}, \pi / 6]$.

In order to show that $\mathfrak{F}(\tilde{c})>\mathfrak{F}(c)$, thus proving (3.15) by contradiction, we need again to estimate the difference between $A_{n}^{2}+B_{n}^{2}$ and $\widetilde{A}_{n}^{2}+\widetilde{B}_{n}^{2}$.

Let us start with $n=3$. From (3.17) we get

$$
\begin{aligned}
\widetilde{A}_{3}-A_{3} & =\int_{\theta^{*}}^{\pi / 6} \sin (3 \theta)(\tilde{c}(\theta)-c(\theta)) d \theta \\
& \geq \varepsilon \int_{0 \vee \theta_{-}}^{\pi / 6} \sin (3 \theta) d \theta+\frac{4}{3} \varepsilon \int_{0 \wedge \theta_{-}}^{0} \sin (3 \theta) d \theta+o(\varepsilon) \\
& \geq \varepsilon \int_{0}^{\pi / 6} \sin (3 \theta) d \theta+\frac{4}{3} \varepsilon \int_{-\hat{\theta}}^{0} \sin (3 \theta) d \theta+o(\varepsilon)=\frac{\varepsilon}{9}(4 \cos (3 \hat{\theta})-1)+o(\varepsilon),
\end{aligned}
$$

so that

$$
\begin{aligned}
\widetilde{A}_{3}^{2}+\widetilde{B}_{3}^{2} & \geq \widetilde{A}_{3}^{2} \geq A_{3}^{2}+\frac{2}{9} A_{3}(4 \cos (3 \hat{\theta})-1) \varepsilon+o(\varepsilon) \\
& =A_{3}^{2}+B_{3}^{2}+\frac{2}{9} A_{3}(4 \cos (3 \hat{\theta})-1) \varepsilon+o(\varepsilon) .
\end{aligned}
$$

Let us now consider the coefficients with $n \geq 5$. Defining $\varphi_{n}$ as in (3.13) and arguing as before, we have

$$
\begin{aligned}
\sqrt{\widetilde{A}_{n}^{2}+\widetilde{B}_{n}^{2}} & \geq \int_{-\pi / 2}^{\pi / 2} \tilde{c}(\theta) \sin \left(n \theta+\varphi_{n}\right) d \theta \\
& =\sqrt{A_{n}^{2}+B_{n}^{2}}+\int_{\theta_{-}}^{\pi / 6}(\tilde{c}(\theta)-c(\theta)) \sin \left(n \theta+\varphi_{n}\right) d \theta+o(\varepsilon) .
\end{aligned}
$$

Divide the interval $\left[\theta_{-}, \pi / 6\right]$ into subintervals where the $\operatorname{sign}$ of $\sin \left(n \theta+\varphi_{n}\right)$ is constant. Clearly, all these subintervals have length $\pi / n$ except possibly the first and the last one. Recall also that

$$
\int_{0}^{\pi / n} \sin (n \theta) d \theta=\frac{2}{n}
$$

Using (3.17), a rough estimate from below of $\sqrt{\widetilde{A}_{n}^{2}+\widetilde{B}_{n}^{2}}-\sqrt{A_{n}^{2}+B_{n}^{2}}$ could be found by estimating $\tilde{c}(\theta)-c(\theta)$ with $\varepsilon$ (resp. $4 \varepsilon / 3)$ where $\sin \left(n \theta+\varphi_{n}\right)$ is positive (resp. negative). Unfortunately, this estimate is not sharp enough to imply $\mathfrak{F}(\tilde{c})>\mathfrak{F}(c)$. Therefore, we need to perform more careful estimates.

We start with $n=5,7$. In this case $n(\hat{\theta}+\pi / 6) \leq 2 \pi$, so that inside $\left[\theta_{-}, \pi / 6\right]$ the set where $\sin \left(n \theta+\varphi_{n}\right)$ is negative can be either a single interval of length up to $\pi / n$, or two intervals with sum of lengths less than $\pi / n$. Thus, an estimate from below for $\widetilde{A}_{n}^{2}+\widetilde{B}_{n}^{2}$ can be obtained by assuming that the worst possible case occurs, i.e., that inside $\left[\theta_{-}, \pi / 6\right]$ there is an interval $I$ of length $\pi / n$ where $\sin \left(n \theta+\varphi_{n}\right)$ is negative and that in this interval 
$\tilde{c}-c$ attains the maximum possible value $4 \varepsilon / 3$. Therefore, recalling (3.19) and (3.20), we get the estimate

$$
\begin{aligned}
\sqrt{\widetilde{A}_{n}^{2}+\widetilde{B}_{n}^{2}}-\sqrt{A_{n}^{2}+B_{n}^{2}} & \geq \int_{I}(\tilde{c}(\theta)-c(\theta)) \sin \left(n \theta+\varphi_{n}\right) d \theta+o\left(\varepsilon^{2}\right) \\
& \geq-\frac{8}{3 n} \varepsilon+o\left(\varepsilon^{2}\right),
\end{aligned}
$$

where we have neglected the positive integral over $\left[\theta_{-}, \pi / 6\right] \backslash I$.

Finally, let us consider the case $n \geq 9$. The interval $J=\left[\theta_{-}, \pi / 6\right]$ is divided into $N$ subintervals $J_{i}, 1 \leq i \leq N$, and to get an estimate of $\sqrt{\widetilde{A}_{n}^{2}+\widetilde{B}_{n}^{2}}$ we consider again the worst possible case that occurs when $N$ is odd and the $\operatorname{sign}$ of $\sin \left(n \theta+\varphi_{n}\right)$ is negative in $J_{i}$ for odd $i$. As we said before, it would be too rough to estimate $\tilde{c}-c$ from below by $\varepsilon$ in all $J_{i}$ with even $i$ and from above by $4 \varepsilon / 3$ in all $J_{i}$ with odd $i$, since this would not be enough to establish inequality (3.11). To get a better estimate, recall that by (3.16) and by the definition of $\theta_{-}$we have $\tilde{c}(\theta)=c(\theta+\varepsilon)$ and $c^{\prime}(\theta)=1+c^{2}(\theta)$ in $J$. Thus, for all $\theta \in J$,

$$
\left|\tilde{c}(\theta)-c(\theta)-\varepsilon\left(1+c^{2}(\theta)\right)\right| \leq \frac{4 \sqrt{3}}{9} \varepsilon^{2} .
$$

Let us now observe that $c$ is increasing in $J$. Therefore, $1+c^{2}$ cannot freely oscillate between 1 and 4/3. Indeed, either $1+c^{2}$ is monotone in the whole interval $J$ (which happens if $c$ has constant sign in $J$ ), or there exists $\bar{\eta} \in J$ such that $c(\bar{\eta})=0$, hence $1+c^{2}$ is decreasing in $J \cap\{\theta \leq \bar{\eta}\}$ and increasing in $J \cap\{\theta \geq \bar{\eta}\}$. As a consequence, if $i$ is an even number such that $1+c^{2}$ is decreasing in $J_{i} \cup J_{i+1}$, from (3.22) we have

$$
\int_{J_{i} \cup J_{i+1}}(\tilde{c}(\theta)-c(\theta)) \sin \left(n \theta+\varphi_{n}\right) d \theta \geq-\frac{4 \sqrt{3}}{9} \varepsilon^{2}\left|J_{i} \cup J_{i+1}\right| ;
$$

similarly, if $i$ is even and $1+c^{2}$ is increasing in $J_{i-1} \cup J_{i}$, we have again

$$
\int_{J_{i-1} \cup J_{i}}(\tilde{c}(\theta)-c(\theta)) \sin \left(n \theta+\varphi_{n}\right) d \theta \geq-\frac{4 \sqrt{3}}{9} \varepsilon^{2}\left|J_{i-1} \cup J_{i}\right| .
$$

Notice that the union of all the sets of the type $J_{i} \cup J_{i+1}$ or of the type $J_{i-1} \cup J_{i}$ considered above does not cover the whole interval $J$ and so we still have to estimate the integral in the complement of this union with respect to $J$. Denote this remaining set by $H$. Notice that three possible cases may occur: either $1+c^{2}$ is monotone in $J$, or $1+c^{2}$ is not monotone in $J$ and $\sin \left(n \bar{\eta}+\varphi_{n}\right) \geq 0$, or $1+c^{2}$ is not monotone in $J$ and $\sin \left(n \bar{\eta}+\varphi_{n}\right) \leq 0$. Correspondingly, the worst possible situations are:

- $H$ is a single interval $J_{i}$, containing one endpoint of $J$, with $i$ odd;

- $H$ is the union of two intervals $J_{i}$ with $i$ odd, each of them containing one endpoint of $J$, and one interval $J_{j}$ with $j$ even, containing $\bar{\eta}$;

- $H$ is the union of two intervals $J_{i}$ with $i$ odd, each of them containing one endpoint of $J$, a third interval $J_{i}$ with $i$ odd, containing $\bar{\eta}$, and two intervals $J_{j}$ adjacent to the latter, with $j$ even. 
Estimating $\tilde{c}(\theta)-c(\theta)$ by $\varepsilon$ in the intervals $J_{i}$ with $i$ even and by $4 \varepsilon / 3$ in the intervals $J_{j}$ with $j$ odd (which is of course admissible), we get, in all the three cases above,

$$
\begin{aligned}
\int_{J}(\tilde{c}(\theta)-c(\theta)) \sin \left(n \theta+\varphi_{n}\right) d \theta & \geq \int_{H}(\tilde{c}(\theta)-c(\theta)) \sin \left(n \theta+\varphi_{n}\right) d \theta-\frac{4 \sqrt{3}}{9} \varepsilon^{2}|J| \\
& \geq-\frac{4}{n} \varepsilon-\frac{4 \sqrt{3}}{9} \varepsilon^{2}|J|
\end{aligned}
$$

From this estimate and (3.19) we then obtain, for all $n \geq 9$,

$$
\sqrt{\widetilde{A}_{n}^{2}+\widetilde{B}_{n}^{2}}-\sqrt{A_{n}^{2}+B_{n}^{2}} \geq-\frac{4}{n} \varepsilon-\frac{4 \sqrt{3}}{9} \varepsilon^{2}|J| .
$$

This estimate is worse than (3.21), valid for $n=5,7$, but still enough to get the inequality $\mathfrak{F}(\tilde{c})>\mathfrak{F}(c)$ and thus to complete the proof of (3.15) by contradiction. Indeed, from (3.21) and (3.23), recalling the estimate (3.6), we get

$$
\begin{aligned}
\sum_{\substack{n \text { odd } \\
n \geq 5}} \frac{\widetilde{A}_{n}^{2}+\widetilde{B}_{n}^{2}-A_{n}^{2}-B_{n}^{2}}{n^{2}-1} & \geq-\sum_{n=5,7} \frac{16 \varepsilon}{3 n} \frac{\sqrt{A_{n}^{2}+B_{n}^{2}}}{n^{2}-1}-\sum_{\substack{n \text { odd } \\
n \geq 9}} \frac{8 \varepsilon}{n} \frac{\sqrt{A_{n}^{2}+B_{n}^{2}}}{n^{2}-1}+O\left(\varepsilon^{2}\right) \\
& \geq-\frac{32 \varepsilon}{9}\left(\sum_{n=5,7} \frac{4}{n^{2}\left(n^{2}-1\right)}+\sum_{\substack{n \text { odd } \\
n \geq 9}} \frac{6}{n^{2}\left(n^{2}-1\right)}\right)+O\left(\varepsilon^{2}\right)
\end{aligned}
$$

On the other hand, using (3.18) and the estimate (3.7),

$$
\frac{\widetilde{A}_{3}^{2}+\widetilde{B}_{3}^{2}-A_{3}^{2}-B_{3}^{2}}{8} \geq \frac{\alpha}{36}(4 \cos (3 \hat{\theta})-1) \varepsilon+O\left(\varepsilon^{2}\right),
$$

where $\alpha$ is as in (3.8). Thus, we can conclude that $\mathfrak{F}(\tilde{c})>\mathfrak{F}(c)$, at least for $\varepsilon$ small enough, provided

$$
\frac{\alpha}{36}(4 \cos (3 \hat{\theta})-1)>\frac{32}{9}\left(\sum_{n=5,7} \frac{4}{n^{2}\left(n^{2}-1\right)}+\sum_{\substack{n \text { odd } \\ n \geq 9}} \frac{6}{n^{2}\left(n^{2}-1\right)}\right) .
$$

To check whether this inequality is true one has to estimate the left and the right hand sides from below and from above respectively. Concerning the right hand side, one needs, as in Step I and in the proof of Lemma 3.8, to estimate the sum by taking the terms up to $n=15$ and then arguing as in (3.9) for the remaining part. Concerning the left hand side, instead, one has only to recall the definition of $\hat{\theta}$ and the estimate (3.7) for $\alpha$. In this way, the desired inequality follows from

$$
38.8 \cdot 10^{-3}>36.8 \cdot 10^{-3}
$$


Step III. Conclusion.

Arguing as in Step I we may conclude that there exist three angles $\theta_{1} \in[\hat{\theta}, \pi / 3-\hat{\theta}]$, $\theta_{2} \in[\pi / 3+\hat{\theta}, 2 \pi / 3-\hat{\theta}]$ and $\theta_{3} \in[2 \pi / 3+\hat{\theta}, \pi-\hat{\theta}]$ such that

$$
c^{\prime}(\theta)= \begin{cases}1+c^{2}(\theta) & \text { in }\left[\hat{\theta}, \theta_{1}\right], \\ -\left(1+c^{2}(\theta)\right) & \text { in }\left[\theta_{1}, \pi / 3-\hat{\theta}\right] \cup\left[\pi / 3+\hat{\theta}, \theta_{2}\right], \\ 1+c^{2}(\theta) & \text { in }\left[\theta_{2}, 2 \pi / 3-\hat{\theta}\right] \cup\left[2 \pi / 3+\hat{\theta}, \theta_{3}\right], \\ -\left(1+c^{2}(\theta)\right) & \text { in }\left[\theta_{3}, \pi-\hat{\theta}\right] .\end{cases}
$$

If we define $\tilde{c}$ to be the reflection of $c$ with respect to $\pi / 6$, i.e. $\tilde{c}(\theta)=c(\pi / 3-\theta)$, it is clear that $\tilde{c}$ is still a weakly admissible, optimal function, whose Fourier coefficients $\widetilde{A}_{3}$ and $\widetilde{B}_{3}$ satisfy $\widetilde{A}_{3}=A_{3}>0, \widetilde{B}_{3}=-B_{3}=0$. With this observation in mind, it is clear that we may assume with no loss of generality that $\theta_{1} \geq \pi / 6$. Hence, by Step II we have $c^{\prime}(\theta)=1+c^{2}(\theta)$ in $\left[\theta_{3}-\pi, \theta_{1}\right]$ (see (3.24) and recall that $\left.c(\pi+\theta)=-c(\theta)\right)$.

Consider now $\theta_{2}$. There are two possible cases.

If $\theta_{2} \geq \pi / 2$, the obvious variant of Step II in the interval $[\pi / 3,2 \pi / 3]$ yields $c^{\prime}(\theta)=$ $-\left(1+c^{2}(\theta)\right)$ in $\left[\theta_{1}, \theta_{2}\right]$. Since $c(\theta)$ in this interval coincides with a suitable translation of $\tan \theta$, by the $L^{\infty}$ estimate (3.1) we have $\theta_{2}-\theta_{1} \leq \pi / 3$, and for the same reason $\theta_{1}-\left(\theta_{3}-\pi\right) \leq \pi / 3$. Therefore, $\theta_{3}-\theta_{2} \geq \pi / 3$ and so, since $\theta_{2} \geq \pi / 2$, we have $\theta_{3} \geq 5 \pi / 6$. Then Step II again gives $c^{\prime}(\theta)=1+c^{2}(\theta)$ in $\left[\theta_{2}, \theta_{3}\right]$, concluding the proof.

Otherwise, if $\theta_{2}<\pi / 2$, arguing exactly as before we have $c^{\prime}(\theta)=1+c^{2}(\theta)$ in $\left[\theta_{2}, \theta_{3}\right]$. Then the $L^{\infty}$ estimate gives $\theta_{1}-\left(\theta_{3}-\pi\right) \leq \pi / 3$ and $\theta_{3}-\theta_{2} \leq \pi / 3$. Therefore, $\theta_{2}-\theta_{1} \geq \pi / 3$, which contradicts the assumption $\theta_{1} \geq \pi / 6$ and $\theta_{2}<\pi / 2$.

Proof of Theorem 4. Let $c$ be a weakly admissible, optimal function. Up to a rotation, we may assume that $A_{3}>0$ and $B_{3}=0$. Let $\theta_{1}, \theta_{2}$ and $\theta_{3}$ be as in Lemma 3.9. Using the $L^{\infty}$ estimate (3.1) as in Step III of the lemma above we have

$$
\theta_{2}-\theta_{1} \leq \pi / 3, \quad \theta_{3}-\theta_{2} \leq \pi / 3, \quad\left(\pi+\theta_{1}\right)-\theta_{3} \leq \pi / 3,
$$

so that the three inequalities must necessarily be equalities. This concludes the proof.

\section{Proof of Theorem 3}

To prove Theorem 3, we will take an optimal function $\bar{c}$, which is now a function maximizing the perimeter (such a function exists by Corollary 3.3), and we consider the different intervals in which it has constant sign. In particular, the essential point is to show that there are exactly three such intervals, each of length $\pi / 3$. In fact, it is quite reasonable that short intervals are not convenient to maximize the perimeter, which is the integral of $\sqrt{1+c^{2}}$, due to the constraint $\left|c^{\prime}\right| \leq 1+c^{2}$. The argument to exclude large intervals is much more delicate, and ultimately relies on the $L^{\infty}$ estimate of Lemma 3.6. In Section 4.1 below we collect some preliminary results and tools, then in Sections 4.2, 4.3 and 4.4 we consider the cases where there are no intervals strictly larger than $\pi / 3$, just one interval, or two intervals, respectively. The proof of the result will then be an immediate consequence. 


\subsection{Preliminary results}

Let us start with some notation. Given any function $c:[0, \pi] \rightarrow \mathbb{R}$ and any Borel subset $I \subseteq[0, \pi]$, we define

$$
P(c):=\int_{0}^{\pi} \sqrt{1+c^{2}(\theta)} d \theta, \quad P(c ; I)=\int_{I} \sqrt{1+c^{2}(\theta)} d \theta .
$$

In particular notice that, if $c: \mathbb{S}^{1} \rightarrow \mathbb{R}$ is an admissible function, and $C$ is the corresponding Zindler set, then by formula (2.13) we have

$$
\text { Perimeter }(C)=2 P(c),
$$

so that the problem of maximizing the perimeter in the class of convex Zindler sets with bisecting chords of length 2 is equivalent to maximizing $P(c)$ among all admissible functions $c$, i.e., satisfying (2.9).

We now give a useful definition: let $\mathfrak{M}:[0, \pi / 3] \rightarrow \mathbb{R}$ be defined by

$$
\mathfrak{M}(x):=2 \ln \left(\frac{1+\sin (x / 2)}{\cos (x / 2)}\right) .
$$

First of all, one may easily observe that $\mathfrak{M}$ is convex and

$$
\mathfrak{M}^{\prime}(x)=\frac{1}{\cos (x / 2)} .
$$

The interest of this function is due to the observation that $\mathfrak{M}(x)$ is the maximum possible perimeter that an admissible function may have in an interval of length $x$ as shown below.

Lemma 4.1 (Characterization of $\mathfrak{M})$. Let $c:[0, \pi] \rightarrow \mathbb{R}$ be a function such that $\left|c^{\prime}\right| \leq$ $1+c^{2}$, and let $I=(a, b) \subseteq[0, \pi]$ be an interval of length at most $\pi / 3$ for which $c(a)=c(b)=0$. Then

$$
P(c ; I) \leq \mathfrak{M}(|I|),
$$

and equality holds if and only if

$$
c(\theta)= \pm \tan (\operatorname{dist}(\theta, \partial I)) \quad \forall \theta \in I .
$$

Proof. Keeping in mind formula (4.1) and the constraint $\left|c^{\prime}\right| \leq 1+c^{2}$, it is clear that $P(c ; I)$ is maximal if and only if $c$ coincides with (4.5) inside $I$. The fact that the perimeter in that case is exactly $\mathfrak{M}(|I|)$ is then an elementary calculation.

We now present a technical result whose statement may appear obscure, but which in fact gives a very strong information on the possible structure of maximizing functions, as will be clear in the proof of Lemma 4.3 below (see Step II). 
Lemma 4.2. Let $\pi / 6 \leq a<b<\pi$, and let $c:[0, \pi] \rightarrow \mathbb{R}$ be a function with $\|c\|_{L^{\infty}} \leq$ $\tan (\pi / 6), c>0$ in $[a, b], c^{\prime}=-\left(1+c^{2}\right)$ in $(a, b)$ and $c^{\prime}=1+c^{2}$ in a left neighborhood of $a$ and in a right neighborhood of $b$. Then, for any $\varepsilon$ such that $|\varepsilon|$ is small enough, there exist a continuous function $c_{\varepsilon}:[0, \pi] \rightarrow \mathbb{R}$ and an interval $\left(a_{\varepsilon}, b_{\varepsilon}\right)$ such that $a_{\varepsilon} \rightarrow a$ and $b_{\varepsilon} \rightarrow b$ as $\varepsilon \rightarrow 0$, and the following holds. The function $c_{\varepsilon}$ coincides with $c$ in $[0, \pi] \backslash\left(\left(a_{\varepsilon}, b_{\varepsilon}\right) \cup(a, b)\right)$, and

$$
\begin{gathered}
c_{\varepsilon}^{\prime}=-\left(1+c_{\varepsilon}^{2}\right) \quad \text { in }\left(a_{\varepsilon}, b_{\varepsilon}\right), \quad c_{\varepsilon}^{\prime}=1+c_{\varepsilon}^{2} \quad \text { in }(a, b) \backslash\left(a_{\varepsilon}, b_{\varepsilon}\right) ; \\
\int_{0}^{\pi} c_{\varepsilon}(\theta) \sin \theta d \theta=\int_{0}^{\pi} c(\theta) \sin \theta d \theta+\varepsilon ;
\end{gathered}
$$

moreover, the Taylor expansion

$$
P\left(c_{\varepsilon}\right)=P(c)+A \varepsilon+B \varepsilon^{2}+o\left(\varepsilon^{2}\right)
$$

holds with two constants $A$ and $B$ not depending on $\varepsilon$, and with $B>0$.

Proof. Given a small $\varepsilon$, we take a constant $\delta=\delta(\varepsilon)$, to be specified later, and we define the function $c_{\varepsilon}$ as the "translation" by $\delta$ of the function $c$, as in Figure 12. To be more precise, if $\varepsilon>0$ as in the figure, then $\delta>0$ and $c_{\varepsilon}$ equals $c$ outside $(a, b+\delta / 2)$ and has $c_{\varepsilon}^{\prime}=1+c_{\varepsilon}^{2}$ in $(a, a+\delta / 2)$ and $c_{\varepsilon}^{\prime}=-\left(1+c_{\varepsilon}^{2}\right)$ in $(a+\delta / 2, b+\delta / 2)$. On the other hand, if $\varepsilon<0$ then the situation is completely symmetric, namely $\delta<0, c_{\varepsilon}$ equals $c$ outside $(a+\delta / 2, b)$ and has $c_{\varepsilon}^{\prime}=-\left(1+c_{\varepsilon}^{2}\right)$ in $(a+\delta / 2, b+\delta / 2)$ and $c_{\varepsilon}^{\prime}=1+c_{\varepsilon}^{2}$ in $(b+\delta / 2, b)$.

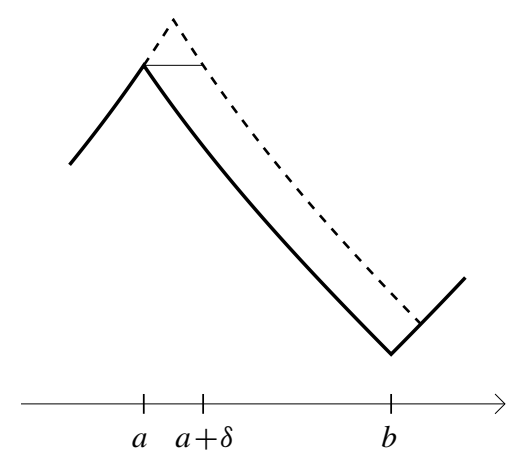

Fig. 12. The functions $c$ (solid) and $c_{\varepsilon}$ (dashed) in Lemma 4.2.

Notice that

$$
c_{\varepsilon}(\theta)=c(\theta-\delta) \quad \forall \theta \in(a+\delta, b+\delta / 2)
$$

if $\varepsilon>0$, while

$$
c_{\varepsilon}(\theta)=c(\theta-\delta) \quad \forall \theta \in(a+\delta / 2, b+\delta)
$$

if $\varepsilon<0$. To get the conclusion, we thus have to select $\delta$ in such a way that (4.6) holds, and then check that (4.7) is true with a strictly positive constant $B$. 
Step I. The relation between $\delta$ and $\varepsilon$.

By the definition of $c_{\varepsilon}$, it is clear that

$$
\int_{0}^{\pi} c_{\varepsilon}(\theta) \sin \theta d \theta-\int_{0}^{\pi} c(\theta) \sin \theta d \theta
$$

is positive if and only if so is $\delta$, and moreover it is a strictly increasing function of $\delta$. Provided $|\varepsilon| \ll 1$, there is a unique $\delta=\delta(\varepsilon)$ which makes (4.6) true. We now give the expression of $\delta$ in terms of $\varepsilon$ up to the second order. Let us start by considering the case $\varepsilon>0$, depicted in Figure 12: we start by rewriting (4.6) as

$$
\begin{aligned}
\varepsilon= & \int_{a}^{b+\delta / 2}\left(c_{\varepsilon}(\theta)-c(\theta)\right) \sin \theta d \theta \\
= & \int_{a}^{a+\delta} c_{\varepsilon}(\theta) \sin \theta d \theta+\int_{a}^{b-\delta / 2} c(\theta)(\sin (\theta+\delta)-\sin \theta) d \theta-\int_{b-\delta / 2}^{b+\delta / 2} c(\theta) \sin \theta d \theta \\
= & \int_{a}^{a+\delta} c_{\varepsilon}(\theta) \sin \theta d \theta-\int_{b-\delta / 2}^{b+\delta / 2} c(\theta) \sin \theta d \theta \\
& +\int_{a}^{b-\delta / 2} c(\theta)\left(\delta \cos \theta-\frac{1}{2} \delta^{2} \sin \theta\right) d \theta+o\left(\delta^{2}\right) .
\end{aligned}
$$

Let us now calculate more precisely the terms of the last line in (4.9). With regard to the first one, notice that for all $0 \leq t \leq \delta / 2$ one has

$$
c_{\varepsilon}(a+t)=c_{\varepsilon}(a+\delta-t)=c(a)+t\left(1+c(a)^{2}\right)+o(\delta),
$$

so that

$$
\begin{aligned}
& \int_{a}^{a+\delta} c_{\varepsilon}(\theta) \sin \theta d \theta \\
& \quad=\int_{t=0}^{\delta / 2}\left(c(a)+t\left(1+c^{2}(a)\right)+o(\delta)\right)(\sin (a+t)+\sin (a+\delta-t)) d t \\
&=\delta c(a) \sin a+\frac{\delta^{2}}{4}\left(1+c^{2}(a)\right) \sin a+\frac{\delta^{2}}{2} c(a) \cos a+o\left(\delta^{2}\right) .
\end{aligned}
$$

The second integral of the last line in (4.9) can be estimated, as before and minding that $c(b+t)=c(b-t)$ for $0 \leq t \leq \delta / 2$, as

$$
\begin{aligned}
\int_{b-\delta / 2}^{b+\delta / 2} c(\theta) \sin \theta d \theta & =\int_{t=0}^{\delta / 2}\left(c(b)+t\left(1+c^{2}(b)\right)+o(\delta)\right)(\sin (b+t)+\sin (b-t)) d t \\
& =\delta c(b) \sin b+\frac{\delta^{2}}{4}\left(1+c^{2}(b)\right) \sin b+o\left(\delta^{2}\right)
\end{aligned}
$$


Finally, the last integral in (4.9) is

$$
\begin{array}{rl}
\int_{a}^{b-\delta / 2} & c(\theta)\left(\delta \cos \theta-\frac{1}{2} \delta^{2} \sin \theta\right) d \theta \\
= & \delta \int_{a}^{b} c(\theta) \cos \theta d \theta-\frac{\delta^{2}}{2} c(b) \cos b-\frac{\delta^{2}}{2} \int_{a}^{b} c(\theta) \sin \theta d \theta+o\left(\delta^{2}\right) .
\end{array}
$$

Putting (4.10)-(4.12) into (4.9), and recalling that $c^{\prime}=-\left(1+c^{2}\right)$ in $(a, b)$, one finds

$$
\begin{aligned}
\varepsilon= & \delta\left(c(a) \sin a-c(b) \sin b+\int_{a}^{b} c(\theta) \cos \theta d \theta\right)+\frac{\delta^{2}}{4}\left(\left(1+c(a)^{2}\right) \sin a\right. \\
& \left.-\left(1+c^{2}(b)\right) \sin b+2 c(a) \cos a-2 c(b) \cos b-2 \int_{a}^{b} c(\theta) \sin \theta d \theta\right)+o\left(\delta^{2}\right) \\
= & \delta \int_{a}^{b}\left(1+c^{2}(\theta)\right) \sin \theta d \theta+\frac{\delta^{2}}{4} \int_{a}^{b}\left(1+c^{2}(\theta)\right)(\cos \theta+2 c(\theta) \sin \theta) d \theta+o\left(\delta^{2}\right) .
\end{aligned}
$$

We have then a second-order relation between $\varepsilon$ and $\delta$ as desired. If $\varepsilon<0$, one has to perform almost identical calculations, eventually leading again to the expression (4.13). Notice that the first coefficient is strictly positive since so is the integrand. Hence, $\delta$ and $\varepsilon$ are of the same order.

Step II. Taylor expansion of $P\left(c_{\varepsilon}\right)-P(c)$.

To finish the proof, we should find the Taylor expansion of $P\left(c_{\varepsilon}\right)-P(c)$ up to the second order in $\varepsilon$, and check that the coefficient of the second term is strictly positive. However, $c_{\varepsilon}$ is defined in a very simple way in terms of $\delta$, not of $\varepsilon$; therefore, we will now find the expansion of $P\left(c_{\varepsilon}\right)-P(c)$ up to the second order in $\delta$, and then we will recover the expansion in terms of $\varepsilon$ by (4.13). Also in this case we show here the calculations when $\varepsilon>0$. The case $\varepsilon<0$ is similar and leads to the same formula.

Since the integral defining the perimeter is non-local, by (4.8) we have

$$
P\left(c_{\varepsilon} ;(a+\delta, b+\delta / 2)\right)=P(c ;(a, b-\delta / 2)) .
$$

Therefore, by (4.1) and the properties of $c$ and $c_{\varepsilon}$ we can evaluate

$$
\begin{aligned}
& P\left(c_{\varepsilon}\right)-P(c)=P\left(c_{\varepsilon} ;(a, a+\delta)\right)-P(c ;(b-\delta / 2, b+\delta / 2)) \\
& =2 \int_{0}^{\delta / 2} \sqrt{1+c_{\varepsilon}^{2}(a+t)} d t-2 \int_{0}^{\delta / 2} \sqrt{1+c^{2}(b+t)} d t \\
& =2 \int_{0}^{\delta / 2}\left(\sqrt{1+c^{2}(a)}-\sqrt{1+c^{2}(b)}+t c(a) \sqrt{1+c^{2}(a)}-t c(b) \sqrt{1+c^{2}(b)}\right) d t+o\left(\delta^{2}\right) \\
& =\delta\left(\sqrt{1+c^{2}(a)}-\sqrt{1+c^{2}(b)}\right)+\frac{\delta^{2}}{4}\left(c(a) \sqrt{1+c^{2}(a)}-c(b) \sqrt{1+c^{2}(b)}\right)+o\left(\delta^{2}\right) \\
& =\delta \int_{a}^{b} c(\theta) \sqrt{1+c^{2}(\theta)} d \theta+\frac{\delta^{2}}{4} \int_{a}^{b} \sqrt{1+c^{2}(\theta)}\left(1+2 c^{2}(\theta)\right) d \theta+o\left(\delta^{2}\right)
\end{aligned}
$$




\section{Step III. Conclusion.}

Putting together (4.13) and (4.14), we can now find the expansion of $D=P\left(c_{\varepsilon}\right)-P(c)$ in terms of $\varepsilon$. Indeed, keep in mind that, when

$$
\varepsilon=\alpha \delta+\tilde{\alpha} \delta^{2}+o\left(\delta^{2}\right), \quad D=\beta \delta+\tilde{\beta} \delta^{2}+o\left(\delta^{2}\right)
$$

and $\alpha>0$, then

$$
D=\frac{\beta}{\alpha} \varepsilon+\frac{1}{\alpha^{3}}(\alpha \tilde{\beta}-\tilde{\alpha} \beta) \varepsilon^{2}+o\left(\varepsilon^{2}\right) .
$$

In our case, then, we have the validity of the Taylor expansion (4.7) with

$$
\begin{aligned}
A & =\frac{\int c(\theta) \sqrt{1+c^{2}(\theta)}}{\int\left(1+c^{2}(\theta)\right) \sin \theta}, \\
B & =\frac{\int\left(1+c^{2}\right) \sin \theta \int \sqrt{1+c^{2}}\left(1+2 c^{2}\right)-\int\left(1+c^{2}\right)(\cos \theta+2 c \sin \theta) \int c \sqrt{1+c^{2}}}{4\left(\int\left(1+c^{2}\right) \sin \theta\right)^{3}},
\end{aligned}
$$

where all the integrals are over $(a, b)$. Thus, we shall have concluded once we show that

$$
\int_{a}^{b}\left(1+c^{2}\right) \sin \theta \int_{a}^{b} \sqrt{1+c^{2}}\left(1+2 c^{2}\right)>\int_{a}^{b}\left(1+c^{2}\right)(\cos \theta+2 c \sin \theta) \int_{a}^{b} c \sqrt{1+c^{2}}
$$

Recall now that, given four functions $f, g, \tilde{f}, \tilde{g}$ on the same interval, to ensure the validity of $\int f \int g>\int \tilde{f} \int \tilde{g}$ it is not enough to check that for all $x$ one has $f(x) g(x)>$ $\tilde{f}(x) \tilde{g}(x)$, but of course it is enough to check that for all $x$ and $y$ one has $f(x) g(y)>$ $\tilde{f}(x) \tilde{g}(y)$. We aim then to prove that for all $\theta, \varphi \in(a, b)$ one has

$$
\begin{aligned}
\left(1+c^{2}(\theta)\right) \sin \theta \cdot \sqrt{1+c^{2}(\varphi)} & \left(1+2 c^{2}(\varphi)\right) \\
> & \left(1+c^{2}(\theta)\right)(\cos \theta+2 c(\theta) \sin \theta) c(\varphi) \sqrt{1+c^{2}(\varphi)},
\end{aligned}
$$

which is equivalent to

$$
\sin \theta \cdot(1+2 c(\varphi)(c(\varphi)-c(\theta)))>c(\varphi) \cos \theta .
$$

Since $\|c\|_{L^{\infty}} \leq \tan (\pi / 6)=\sqrt{3} / 3$ and $c$ is positive in $(a, b) \subseteq(\pi / 6, \pi)$, the left term is always positive, so the inequality (4.15) is trivially true if $\theta \geq \pi / 2$. Hence, we have to show that

$$
\tan \theta>\frac{c(\varphi)}{1+2 c(\varphi)(c(\varphi)-c(\theta))}
$$

for all $\varphi, \theta \in(a, b)$ with $\theta<\pi / 2$. As $\theta>a \geq \pi / 6$, to get the full validity of (4.15) and then to conclude it is enough to verify that for all $\theta, \varphi \in(a, b)$,

$$
\frac{\sqrt{3}}{3}>\frac{c(\varphi)}{1+2 c(\varphi)(c(\varphi)-c(\theta))},
$$


which in turn follows from the fact that

$$
\max _{x, y \in[0, \sqrt{3} / 3]} \frac{y}{1+2 y(y-x)}=\frac{\sqrt{3}}{3}
$$

and the sup is not reached in the interior of the square $[0, \sqrt{3} / 3]^{2}$.

In Lemma 4.1 we have given the estimate (4.4), which gives a bound on the perimeter inside an interval of length at most $\pi / 3$. The next lemma, instead, gives the bound (4.16) for larger intervals.

Lemma 4.3. Let $I=[\bar{\theta}, \pi-\bar{\theta}]$ with $\pi / 6<\bar{\theta}<\pi / 3$, and let $\tilde{c}:[0, \pi] \rightarrow \mathbb{R}$ be a function satisfying the assumptions (3.3) of Lemma 3.6. Then

$$
P(\tilde{c} ; I)<2 \mathfrak{M}(\pi / 3)-\mathfrak{M}(2 \pi / 3-|I|) .
$$

Proof. The proof is divided into several steps.

Step I. The auxiliary problems.

We will argue by approximation with a sequence of auxiliary problems. To this end, we say that a function $c$ satisfying (3.3) is an $n$-step function if $I \cap\{c>0\}$ is the union of at most $n$ subintervals where $c^{\prime}=1+c^{2}$ and at most $n$ subintervals where $c^{\prime}=-\left(1+c^{2}\right)$. For instance, the function in Figure 13 is a 6-step function, but also an $n$-step function for all $n \geq 6$.

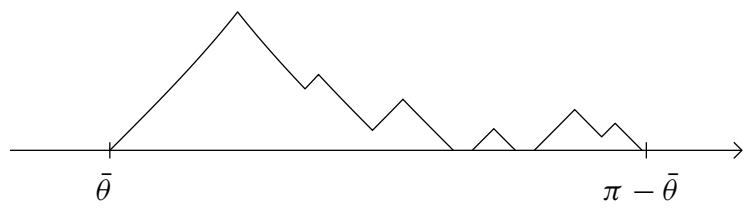

Fig. 13. Example of a 6-step function.

The auxiliary problems are then defined as follows: for any $n \in \mathbb{N}$, find

$$
\begin{array}{r}
\max \{P(c ; I): c:[0, \pi] \rightarrow \mathbb{R}, c \text { satisfies (3.3), } c \text { is an } n \text {-step function, } \\
\left.\qquad=\tilde{c} \text { in }[0, \pi] \backslash I, \int_{I} c(\theta) \sin \theta d \theta \leq \int_{I} \tilde{c}(\theta) \sin \theta d \theta\right\} .
\end{array}
$$

By trivial compactness, each of the problems (4.17) admits a solution $c_{n}$. Moreover, since the function $\tilde{c}$ itself can be approximated with a sequence of $n$-step functions admissible for the problems (4.17), we have $P(\tilde{c} ; I) \leq \liminf P\left(c_{n} ; I\right)$. Therefore, the conclusion will be achieved if we prove that for a generic $n \in \mathbb{N}$ one has

$$
P\left(c_{n} ; I\right) \leq 2 \mathfrak{M}(\pi / 3)-\mathfrak{M}(2 \pi / 3-|I|)-\omega(|I|-\pi / 3),
$$

where $\omega:(0, \pi / 3] \rightarrow \mathbb{R}$ is a strictly positive function, not depending on $n$. 
Let us then fix a number $n$ and prove the estimate. First of all, we denote by $I_{j}$, with $j \geq 1$, all the maximal subintervals of $I$ where $c_{n}$ is strictly positive: for instance, the function in Figure 13 has three such intervals. Moreover, we set $I_{0}=I \backslash \bigcup_{j \geq 1} I_{j}$ : in the following, we will refer to all the $I_{j}$ 's as "subintervals" of $I$, though of course $I_{0}$ may not be an interval. The restriction of $c_{n}$ to each subinterval $I_{j}$ with $j \geq 1$ is clearly still an $n$-step function, but we see now that much more is true.

Step II. On each $I_{j}$ with $j \geq 1$, the function $c_{n}$ is either a 1-step function or a 2-step function.

Suppose for contradiction that for some $I_{j}$ there exist three (or more) disjoint, maximal subintervals of $I_{j}$ where $c_{n}$ is decreasing with maximal slope. We can thus select $\varepsilon$ with $|\varepsilon| \ll 1$ and apply Lemma 4.2 to the first and to the second subinterval with $\varepsilon$ and $-\varepsilon$ respectively (notice that the $L^{\infty}$ bound required by Lemma 4.2 is fulfilled as a consequence of Lemma 3.6). We thus have now a function $c_{n, \varepsilon}$, which equals $c_{n}$ off a small neighborhood of the two subintervals, and which is still admissible for problem (4.17). Indeed, by construction $c_{n, \varepsilon}$ is still an $n$-step function, and moreover

$$
\int_{I} c_{n, \varepsilon}(\theta) \sin \theta d \theta=\int_{I} c_{n}(\theta) \sin \theta d \theta
$$

as a consequence of (4.6), since the integral increases in $\varepsilon$ in the first subinterval and decreases in $\varepsilon$ in the second one. Since $c_{n}$ is optimal for problem (4.17), we deduce that

$$
P\left(c_{n} ; I\right) \geq P\left(c_{n, \varepsilon} ; I\right) \text {. }
$$

Let us now use the Taylor expansion (4.7): there are constants $A_{1}, A_{2}, B_{1}$ and $B_{2}$ such that

$$
P\left(c_{n, \varepsilon} ; I\right)=P\left(c_{n} ; I\right)+\left(A_{1}-A_{2}\right) \varepsilon+\left(B_{1}+B_{2}\right) \varepsilon^{2}+o\left(\varepsilon^{2}\right) .
$$

But this is easily seen to contradict (4.19): indeed, if $A_{1}>A_{2}$ it is enough to take $0<$ $\varepsilon \ll 1$, and if $A_{1}<A_{2}$ to take $-1 \ll \varepsilon<0$, to find a first-order contradiction to (4.19). If $A_{1}=A_{2}$, instead, regardless of the sign of $\varepsilon$ we find a second-order contradiction since $B_{1}$ and $B_{2}$ are both strictly positive. The contradiction concludes this step.

Step III. A bound on the perimeter in the subintervals $I_{j}, j \geq 0$.

We can now give a bound on $P\left(c_{n} ; I_{j}\right)$ for each of the subintervals $I_{j}, j \geq 0$, namely

$$
P\left(c_{n} ; I_{j}\right) \leq \begin{cases}\mathfrak{M}\left(\left|I_{j}\right|\right) & \text { if }\left|I_{j}\right| \leq \pi / 3 \\ 2 \mathfrak{M}(\pi / 3)-\mathfrak{M}\left(2 \pi / 3-\left|I_{j}\right|\right)-\omega(|I|-\pi / 3) & \text { if }\left|I_{j}\right|>\pi / 3\end{cases}
$$

Let us start by assuming $j \geq 1$. The first case is already proved by Lemma 4.1. Concerning the second case, notice that by Step II we already know that $c_{n}$ in $I_{j}$ is a 1 -step function or a 2-step function. We can exclude the first possibility, because in that case one would have

$$
\left\|c_{n}\right\|_{L^{\infty}\left(I_{j}\right)}=\tan \left(\left|I_{j}\right| / 2\right)>\tan (\pi / 6) \geq \tan (\bar{\theta} / 2)
$$


contrary to the $L^{\infty}$ bound given by Lemma 3.6. We know then that in $I_{j}$ the function $c_{n}$ is made up of four parts with maximal slope, the first and third increasing, and the second and fourth decreasing. Again keeping in mind Lemma 3.6, we know that all these four parts are shorter than $\bar{\theta} / 2$ (by the way, this ensures that necessarily $\left|I_{j}\right| \leq 2 \bar{\theta}$ ), and then inside $I_{j}=:(a, b)$ the function $c_{n}$ is pointwise smaller (hence, it has a smaller perimeter) than

$$
\begin{cases}\tan (\theta-a) & \text { for } a \leq \theta \leq a+\bar{\theta} / 2 \\ \tan (a-\theta+\bar{\theta}) & \text { for } a+\bar{\theta} / 2 \leq \theta \leq(a+b) / 2 \\ \tan (\theta-b+\bar{\theta}) & \text { for }(a+b) / 2 \leq \theta \leq b-\bar{\theta} / 2 \\ \tan (b-\theta) & \text { for } b-\bar{\theta} / 2 \leq \theta \leq b\end{cases}
$$

Finally, the perimeter inside $I_{j}=(a, b)$ of this last function is exactly

$$
P\left(c_{n} ; I_{j}\right)=2 \mathfrak{M}(\bar{\theta})-\mathfrak{M}\left(2 \bar{\theta}-\left|I_{j}\right|\right),
$$

as one can check with the same elementary calculation as in Lemma 4.1 or directly by an immediate geometric argument. Now, since $\bar{\theta}>2 \bar{\theta}-\left|I_{j}\right|$, by the strict convexity of $\mathfrak{M}$ (which also ensures the strict subadditivity, as $\mathfrak{M}(0)=0$ ), one finds

$$
\begin{aligned}
2 \mathfrak{M}(\bar{\theta})-\mathfrak{M}\left(2 \bar{\theta}-\left|I_{j}\right|\right) & \leq 2 \mathfrak{M}(\pi / 3)-\mathfrak{M}\left(2 \pi / 3-\left|I_{j}\right|\right)-\widetilde{\omega}(\pi / 3-\bar{\theta}) \\
& =2 \mathfrak{M}(\pi / 3)-\mathfrak{M}\left(2 \pi / 3-\left|I_{j}\right|\right)-\omega(|I|-\pi / 3),
\end{aligned}
$$

so that we have proved both cases of (4.20) for $j \geq 1$.

Consider now $I_{0}$. If $\left|I_{0}\right| \leq \pi / 3$, then

$$
P\left(c_{n} ; I_{0}\right)=\left|I_{0}\right| \leq \mathfrak{M}\left(\left|I_{0}\right|\right) .
$$

Conversely, if $\left|I_{0}\right|>\pi / 3$, then

$$
\begin{aligned}
P\left(c_{n} ; I_{0}\right) & =\left|I_{0}\right|=\pi / 3+\left(\left|I_{0}\right|-\pi / 3\right)=\mathfrak{M}(\pi / 3)-\kappa+\left(\left|I_{0}\right|-\pi / 3\right) \\
& \leq \mathfrak{M}(\pi / 3)-\kappa+\mathfrak{M}\left(\left|I_{0}\right|-\pi / 3\right) \leq 2 \mathfrak{M}(\pi / 3)-\kappa-\mathfrak{M}\left(2 \pi / 3-\left|I_{0}\right|\right),
\end{aligned}
$$

where $\kappa=\mathfrak{M}(\pi / 3)-\pi / 3$ is a strictly positive constant. Hence, (4.20) is proved also in the case $j=0$, so this step is concluded.

Step IV. The case when all subintervals $I_{j}, j \geq 0$, are shorter than $\pi / 3$.

Suppose that all the intervals $I_{j}$ have length at most $\pi / 3$ : then we immediately get (4.18) using the first bound of (4.20) and keeping in mind the strict convexity of $\mathfrak{M}$, since

$$
\begin{aligned}
P\left(c_{n} ; I\right) & =\sum_{j} P\left(c_{n} ; I_{j}\right) \leq \sum_{j} \mathfrak{M}\left(\left|I_{j}\right|\right) \leq \mathfrak{M}(\pi / 3)+\mathfrak{M}(|I|-\pi / 3) \\
& \leq 2 \mathfrak{M}(\pi / 3)-\mathfrak{M}(2 \pi / 3-|I|)-\omega(|I|-\pi / 3) .
\end{aligned}
$$

Step V. The case when a subinterval is strictly longer than $\pi / 3$.

Since the length of $I$ is between $\pi / 3$ and $2 \pi / 3$, there can be at most one subinterval strictly longer than $\pi / 3$, say $I_{l}$. We then use the second and the first bound of (4.20) 
respectively for $I_{l}$ and for all the other subintervals, and again the strict convexity and strict subadditivity of $\mathfrak{M}$, to find

$$
\begin{aligned}
P\left(c_{n} ; I\right) & =\sum_{j} P\left(c_{n} ; I_{j}\right)=P\left(c_{n} ; I_{l}\right)+\sum_{j \neq l} P\left(c_{n} ; I_{j}\right) \\
& \leq 2 \mathfrak{M}(\pi / 3)-\mathfrak{M}\left(2 \pi / 3-\left|I_{l}\right|\right)+\sum_{j \neq l} \mathfrak{M}\left(\left|I_{j}\right|\right)-\omega(|I|-\pi / 3) \\
& \leq 2 \mathfrak{M}(\pi / 3)-\mathfrak{M}\left(2 \pi / 3-\left|I_{l}\right|\right)+\mathfrak{M}\left(\sum_{j \neq l}\left|I_{j}\right|\right)-\omega(|I|-\pi / 3) \\
& \leq 2 \mathfrak{M}(\pi / 3)-\mathfrak{M}\left(2 \pi / 3-\sum_{j}\left|I_{j}\right|\right)-\omega(|I|-\pi / 3) \\
& =2 \mathfrak{M}(\pi / 3)-\mathfrak{M}(2 \pi / 3-|I|)-\omega(|I|-\pi / 3) .
\end{aligned}
$$

Therefore, we have proved (4.18) also in this last case and the proof is complete.

\subsection{Step 0 of the proof: no "large" intervals}

In this section we prove a bound on the perimeter of a function $\bar{c}$ which has all the intervals of constant sign shorter than $\pi / 3$.

Proposition 4.4. Let $\bar{c}: \mathbb{S}^{1} \rightarrow \mathbb{R}$ be an admissible function, i.e., satisfying (2.9) and such that all the intervals where $\bar{c}$ has a constant sign have length at most $\pi / 3$. Then $P(\bar{c}) \leq P(\hat{c})$ and equality holds if and only if $\bar{c}=\hat{c}$ up to a rotation.

Proof. Up to a rotation, we may assume that $\bar{c}(0)=\bar{c}(\pi)=0$. Let then $I_{j}$ be the maximal subinterval of $(0, \pi)$ where $\bar{c}$ has a constant sign. By additivity of the perimeter, one has

$$
P(\bar{c})=\sum P\left(\bar{c} ; I_{j}\right)+P\left(\bar{c} ;[0, \pi] \backslash \bigcup I_{j}\right) .
$$

By Lemma 4.1 , by the fact that $\mathfrak{M}(x) \geq x$, and by the convexity and subadditivity of the functional $\mathfrak{M}$, we directly get

$$
P(\bar{c}) \leq \sum \mathfrak{M}\left(\left|I_{j}\right|\right)+\left(\pi-\sum\left|I_{j}\right|\right) \leq 3 \mathfrak{M}(\pi / 3)=P(\hat{c}) .
$$

Moreover, by the strict convexity of $\mathfrak{M}$ (and the fact that $\mathfrak{M}(x)>x$ when $x>0$ ) the second inequality holds as an equality if and only if there are exactly three intervals $I_{j}$, all of length $\pi / 3$. If this is the case, Lemma 4.1 again implies that the first inequality is an equality if and only if $\bar{c}=\hat{c}$ up to a rotation.

\subsection{Step 1 of the proof: one "large" interval}

In this section we prove a bound on the perimeter of a function $\bar{c}$ for which there exists exactly one interval strictly longer than $\pi / 3$ where $\bar{c}>0$ (then also exactly one large interval where $\bar{c}<0$ ). 
Proposition 4.5. Let $\bar{c}: \mathbb{S}^{1} \rightarrow \mathbb{R}$ be an admissible function such that there exists only one maximal interval I where $\bar{c}>0$ such that $|I|>\pi / 3$. Then $P(\bar{c})<P(\hat{c})$.

Proof. As before, we start by assuming that $\bar{c}(0)=\bar{c}(\pi)=0$. We distinguish two cases.

Case I. $\pi / 3<|I|<2 \pi / 3$.

Let us denote by $I_{j}$ the maximal subintervals of $(0, \pi)$ where $\bar{c}$ has a constant sign and which are different from $I$. Arguing exactly as in the proof of Proposition 4.4, we have

$$
\begin{aligned}
P(\bar{c}) & =P(\bar{c} ; I)+\sum_{j} P\left(\bar{c} ; I_{j}\right)+\left(\pi-|I|-\sum_{j}\left|I_{j}\right|\right) \\
& \leq P(\bar{c} ; I)+\mathfrak{M}(\pi / 3)+\mathfrak{M}(2 \pi / 3-|I|) .
\end{aligned}
$$

We now need to estimate $P(\bar{c} ; I)$. Define $\tilde{c}: \mathbb{S}^{1} \rightarrow \mathbb{R}$ by $\tilde{c}(\theta)=\bar{c}(\theta-\varphi)$ where $\varphi$ is chosen in such a way that $I+\varphi=(\bar{\theta}, \pi-\bar{\theta})$ where

$$
\bar{\theta}=\frac{\pi-|I|}{2} \in(\pi / 6, \pi / 3) .
$$

We are then in a position to use Lemma 4.3, hence (4.16) gives

$$
P(\bar{c} ; I)=P(\tilde{c} ;(\bar{\theta}, \pi-\bar{\theta}))<2 \mathfrak{M}(\pi / 3)-\mathfrak{M}(2 \pi / 3-|I|) .
$$

Inserting this inequality in (4.21), we directly get

$$
P(\bar{c})<3 \mathfrak{M}(\pi / 3)=P(\hat{c}) .
$$

Case II. $|I| \geq 2 \pi / 3$.

In this case, applying Lemma 3.6 to a suitable rotation $\tilde{c}$ of $\bar{c}$ as in Case I, we get

$$
\|\bar{c}\|_{L^{\infty}(I)} \leq \tan \left(\frac{\pi-|I|}{4}\right) .
$$

Thus, denoting again by $I_{j}$ the other intervals of constant sign of $\bar{c}$ in $(0, \pi)$ and arguing as before, we find

$$
\begin{aligned}
P(\bar{c}) & \leq \mathfrak{M}(\pi-|I|)+P(\bar{c} ; I) \leq \mathfrak{M}(\pi-|I|)+|I| \sqrt{1+\tan ^{2}\left(\frac{\pi-|I|}{4}\right)} \\
& \leq \mathfrak{M}(\pi / 3)+2 \pi / 3 \sqrt{1+\tan ^{2}(\pi / 12)}
\end{aligned}
$$

where the last inequality follows from the fact that the function

$$
(2 \pi / 3, \pi) \ni x \mapsto \mathfrak{M}(\pi-x)+x \sqrt{1+\tan ^{2}((\pi-x) / 4)}
$$

is strictly decreasing — which is elementary to check starting from the definition of $\mathfrak{M}$. To conclude, it is enough to check that

$$
\frac{1}{3} \pi \sqrt{1+\tan ^{2}(\pi / 12)}<\mathfrak{M}(\pi / 3),
$$

which is again an elementary calculation. 


\subsection{Step 2 of the proof: two "large" intervals}

In this last section we prove the bound on the perimeter for a function $\bar{c}$ which has two intervals strictly longer than $\pi / 3$ where $\bar{c}>0$ (then also two large intervals where $\bar{c}<0$ ).

Proposition 4.6. Let $\bar{c}: \mathbb{S}^{1} \rightarrow \mathbb{R}$ be an admissible function such that there are two maximal intervals strictly longer than $\pi / 3$ where $\bar{c}>0$. Then $P(\bar{c})<P(\hat{c})$.

Proof. The proof is divided into four parts.

Part I. Preliminary observations and setting the auxiliary problem.

We start by assuming that the two large intervals, say $I_{1}$ and $I_{2}$, are both contained in $(0, \pi)$ and that $I_{1}=\left(0,\left|I_{1}\right|\right)$, which is admissible up to a rotation. Recall that, as seen in the proof of Case II of Proposition 4.5 above, if an admissible function $c$ contains an interval of constant sign larger than $2 \pi / 3$ then $P(c) \leq P(\hat{c})-\delta_{0}$ for a strictly positive constant $\delta_{0}$. Hence, by continuity, there exists a strictly positive constant $\varepsilon_{0} \ll 1$ such that, if the distance between the intervals $I_{1}$ and $I_{2}$ of $\bar{c}$ is smaller than $\varepsilon_{0}$, then $P(\bar{c})<P(\hat{c})$. Thus we may assume without loss of generality that $\operatorname{dist}\left(I_{1}, I_{2}\right) \geq \varepsilon_{0}$.

We now introduce the auxiliary problem that we will use throughout the proof. We denote by $\mathcal{Z}$ the set of functions $c:[0, \pi] \rightarrow \mathbb{R}$ that will be considered for the auxiliary problem, such that:

- $c(0)=c(\pi)=0,\left|c^{\prime}\right| \leq 1+c^{2}, \int_{0}^{\pi} c(\theta) \sin \theta d \theta \leq 0$;

- there exist two open intervals $I_{1}=\left(0,\left|I_{1}\right|\right)$ and $I_{2}$ such that $c \geq 0$ on both $I_{j}, c=0$ on $\partial I_{j}$, and $\left|I_{j}\right|=\pi / 3+\ell_{j}$ with $\ell_{1}, \ell_{2} \geq 0$;

- $\operatorname{dist}\left(I_{1}, I_{2}\right) \geq \varepsilon_{0}$, and $c \leq \tan \left(\pi / 6-\ell_{j} / 4\right)$ in $I_{j}$.

Notice that, due to the last condition, the closed intervals $\bar{I}_{1}$ and $\bar{I}_{2}$ might not be maximal intervals where $c \geq 0$. Moreover, $\bar{c}$ clearly belongs to $\mathcal{Z}$, due to the $L^{\infty}$ estimate given by Lemma 3.6. It is straightforward to notice that $\mathcal{Z}$ is compact with respect to uniform convergence, hence the problem

$$
\max \{P(c): c \in \mathcal{Z}\}
$$

has a solution. We state the following

Claim. Problem (4.23) admits $\hat{c}$ as the unique solution.

The proposition will immediately follow once this Claim is established.

Let us now take a solution $\tilde{c}$ of problem (4.23) and divide the proof of the claim into three distinct cases, depending on whether the intervals $\widetilde{I}_{1}$ and $\widetilde{I}_{2}$ are both of length $\pi / 3$, just one is longer, or both are longer.

Part II. If $\left|\widetilde{I}_{1}\right|=\left|\tilde{I}_{2}\right|=\pi / 3$, then $\tilde{c}=\hat{c}$.

In this case, the intervals of constant sign of $\tilde{c}$ have length at most $\pi / 3$, so arguing exactly as in Proposition 4.4 (where we did not really use the assumption (2.9)) we get $P(\tilde{c}) \leq P(\hat{c})$. On the other hand, since $\hat{c} \in \mathcal{Z}$ the reverse inequality is trivial, and thus 
Proposition 4.4 tells us also that $\tilde{c}=\hat{c}$ up to a rotation. Since $\tilde{c}(0)=0$ and $\tilde{c}$ is positive in $(0, \pi / 3)$, we conclude that $\tilde{c}=\hat{c}$.

Part III. It is not possible that $\left|\widetilde{I}_{1}\right|>\left|\tilde{I}_{2}\right|=\pi / 3$ or $\left|\widetilde{I}_{2}\right|>\left|\widetilde{I}_{1}\right|=\pi / 3$.

Assume that $\left|\widetilde{I}_{1}\right|>\left|\widetilde{I}_{2}\right|=\pi / 3$ (the other case being identical), and denote by $\widetilde{J}_{\widetilde{J}_{1}}$ the interval between $\widetilde{I}_{1}$ and by $\widetilde{I}_{2}$, and $\widetilde{J}_{2}$ the interval between $\widetilde{I}_{2}$ and $\pi$. Notice that $\widetilde{J}_{1}$ has length at least $\varepsilon_{0}$, while $\widetilde{J}_{2}$ is possibly empty. We will prove this part by showing that $P(\tilde{c})<P(\hat{c})$, a contradiction with the optimality of $\tilde{c}$. To do so, we split

$$
\begin{aligned}
P(\tilde{c}) & =P\left(\tilde{c} ; \widetilde{I}_{1}\right)+P\left(\tilde{c} ; \widetilde{J}_{1}\right)+P\left(\tilde{c} ; \widetilde{I}_{2}\right)+P\left(\tilde{c} ; \widetilde{J}_{2}\right) \\
& \leq P\left(\tilde{c} ; \widetilde{I}_{1}\right)+\mathfrak{M}\left(\left|\widetilde{J}_{1}\right|\right)+\mathfrak{M}\left(\left|\widetilde{I}_{2}\right|\right)+\mathfrak{M}\left(\left|\widetilde{J}_{2}\right|\right) \\
& \leq P\left(\tilde{c} ; \widetilde{I}_{1}\right)+\mathfrak{M}(\pi / 3)+\mathfrak{M}\left(\left|\widetilde{J}_{1}\right|+\left|\widetilde{J}_{2}\right|\right) \\
& =P\left(\tilde{c} ; \widetilde{I}_{1}\right)+\mathfrak{M}(\pi / 3)+\mathfrak{M}\left(\pi / 3-\tilde{\ell}_{1}\right),
\end{aligned}
$$

so that the desired contradiction will follow once we show that

$$
P\left(\tilde{c} ; \tilde{I}_{1}\right)+\mathfrak{M}\left(\pi / 3-\tilde{\ell}_{1}\right)<2 \mathfrak{M}(\pi / 3) .
$$

Recalling that $\tilde{c} \leq \tan \left(\pi / 6-\tilde{\ell}_{1} / 4\right)$ on $\widetilde{I}_{1}$ and $\tilde{c}=0$ on $\partial \widetilde{I}_{1}$, we find that, inside $\widetilde{I}_{1}$,

$$
\tilde{c}(\theta) \leq \begin{cases}\tan \theta & \text { for } 0 \leq \theta \leq \pi / 6-\tilde{\ell}_{1} / 4, \\ \tan \left(\pi / 6-\tilde{\ell}_{1} / 4\right) & \text { for } \pi / 6-\tilde{\ell}_{1} / 4 \leq \theta \leq \pi / 6+5 \tilde{\ell}_{1} / 4, \\ \tan \left(\pi / 3+\tilde{\ell}_{1}-\theta\right) & \text { for } \pi / 6+5 \tilde{\ell}_{1} / 4 \leq \theta \leq \pi / 3+\tilde{\ell}_{1},\end{cases}
$$

and thus

$$
P\left(\tilde{c} ; \tilde{I}_{1}\right) \leq \mathfrak{M}\left(\pi / 3-\tilde{\ell}_{1} / 2\right)+\frac{3}{2} \tilde{\ell}_{1} \frac{1}{\cos \left(\pi / 6-\tilde{\ell}_{1} / 4\right)} .
$$

Keeping in mind formula (4.2) for $\mathfrak{M}$, the proof of (4.24) can be reduced to checking that

$$
\begin{array}{r}
2 \ln \left(\frac{1+\sin \left(\pi / 6-\tilde{\ell}_{1} / 4\right)}{\cos \left(\pi / 6-\tilde{\ell}_{1} / 4\right)}\right)+\frac{3}{2} \tilde{\ell}_{1} \frac{1}{\cos \left(\pi / 6-\tilde{\ell}_{1} / 4\right)}+2 \ln \left(\frac{1+\sin \left(\pi / 6-\tilde{\ell}_{1} / 2\right)}{\cos \left(\pi / 6-\tilde{\ell}_{1} / 2\right)}\right) \\
<4 \ln \left(\frac{1+\sin (\pi / 6)}{\cos (\pi / 6)}\right)
\end{array}
$$

and since $0<\tilde{\ell}_{1} \leq \pi / 3-\varepsilon_{0}<\pi / 3$, we will be done if we show that

$$
\phi(x)<0 \quad \text { in }(0, \pi / 12),
$$

where

$$
\begin{aligned}
\phi(x):= & 2 \ln \left(\frac{1+\sin (\pi / 6-x)}{\cos (\pi / 6-x)}\right)+\frac{6 x}{\cos (\pi / 6-x)}+2 \ln \left(\frac{1+\sin (\pi / 6-2 x)}{\cos (\pi / 6-2 x)}\right) \\
& -4 \ln \left(\frac{1+\sin (\pi / 6)}{\cos (\pi / 6)}\right) .
\end{aligned}
$$


Since $\phi(0)=0$, it is enough to check that $\phi^{\prime}(x)<0$ in $(0, \pi / 12)$, that is, recalling also (4.3),

$$
\frac{4-6 x \tan (\pi / 6-x)}{\cos (\pi / 6-x)}-\frac{4}{\cos (\pi / 6-2 x)}<0 \quad \text { in }(0, \pi / 12) .
$$

Since $\tan (\pi / 6-x)>\pi / 6-x$, the previous inequality is certainly true if we show that

$$
\frac{4-6 x(\pi / 6-x)}{\cos (\pi / 6-x)}-\frac{4}{\cos (\pi / 6-2 x)}<0 \quad \text { in }(0, \pi / 12),
$$

which is in turn equivalent to

$$
6 x^{2}-\pi x+4<\frac{4 \cos (\pi / 6-x)}{\cos (\pi / 6-2 x)} \quad \text { in }(0, \pi / 12) .
$$

Summarizing, to deduce this part we have reduced (4.25) to (4.26). Notice that the functions on the two sides of this inequality coincide at $x=0$, therefore to prove (4.26) it is enough to show the corresponding inequality for the derivatives, i.e.,

$$
12 x-\pi<4 \frac{\sin x-\cos (\pi / 6-x) \sin (\pi / 6-2 x)}{\cos ^{2}(\pi / 6-2 x)} \text { in }(0, \pi / 12) .
$$

Since $\sin x>0$ and $\cos (\pi / 6-x)<\cos (\pi / 6-2 x)$, we further reduce to

$$
4 \tan (\pi / 6-2 x)<\pi-12 x \text { in }(0, \pi / 12) .
$$

We finally conclude by observing that the function on the left is strictly convex and the function on the right is affine, and noticing that the inequality holds for $x=0$ while equality holds for $x=\pi / 12$.

Part IV. It is not possible that $\left|\widetilde{I}_{1}\right|,\left|\widetilde{I}_{2}\right|>\pi / 3$.

Let $\widetilde{J}_{1}$ and $\widetilde{J}_{2}$ be the intervals as in Part III. This last part will be the most involved one, so we further subdivide it.

Part IV.1. $\tilde{c}$ is "minimal” in $\widetilde{J}_{1}$ and $\widetilde{J}_{2}$.

Since $\tilde{c}=0$ on $\partial \widetilde{J}_{j}$, by the constraint on $\tilde{c}^{\prime}$ one has the pointwise inequality

$$
|\tilde{c}(\theta)| \leq \tan \left(\operatorname{dist}\left(\theta, \partial \widetilde{J}_{j}\right)\right) \quad \text { in } \widetilde{J}_{j} .
$$

On replacing $\tilde{c}$ with $-\tan \left(\operatorname{dist}\left(\theta, \partial \widetilde{J}_{j}\right)\right)$ the perimeter would increase and the resulting function would still belong to $\mathcal{Z}$. So, we may assume without loss of generality that

Part IV.2. Shape of $\tilde{c}$ in $\tilde{I}_{1}$ and $\tilde{I}_{2}$.

$$
\tilde{c}(\theta)=-\tan \left(\operatorname{dist}\left(\theta, \partial \widetilde{J}_{j}\right)\right) \quad \text { in } \widetilde{J}_{j}
$$

We aim to prove that $\tilde{c}$ decreases with the maximal possible slope in a left neighborhood of the right endpoint of $\widetilde{I}_{1}$, more precisely

$$
\tilde{c}(\theta)=\tan \left(\left|\tilde{I}_{1}\right|-\theta\right) \quad \text { in }\left[\left|\tilde{I}_{1}\right|-\left|\tilde{J}_{1}\right| / 2,\left|\tilde{I}_{1}\right|\right] .
$$


Indeed, let us define

$$
\theta^{-}:=\min \left\{\theta \geq 0: \tilde{c}(\varphi)=\tan \left(\left|\widetilde{I}_{1}\right|-\varphi\right) \text { in }\left[\theta,\left|\widetilde{I}_{1}\right|\right]\right\},
$$

so that (4.27) amounts to $\theta^{-} \leq\left|\tilde{I}_{1}\right|-\left|\widetilde{J}_{1}\right| / 2$. To prove this inequality, fix $0<\varepsilon \ll 1$ and set

$$
\hat{\theta}:=\max \left\{\theta \in \widetilde{I}_{1}: \tilde{c}(\theta)=\tan \left(\left|\tilde{I}_{1}\right|-\varepsilon-\theta\right)\right\} .
$$

Clearly, $\hat{\theta}<\theta^{-}$and $\hat{\theta}=\theta^{-}+o(1)$ as $\varepsilon \rightarrow 0$. We now define the function $c^{*}$, shown in

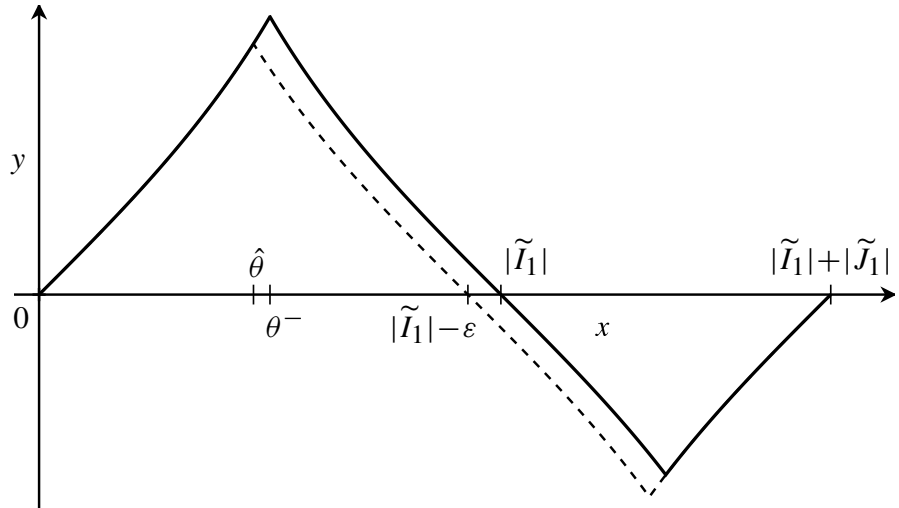

Fig. 14. The functions $\tilde{c}$ (solid) and $c^{*}$ (dashed) in Part IV.2.

Figure 14, as follows:

$$
c^{*}(\theta):= \begin{cases}\tilde{c}(\theta) & \text { if } 0 \leq \theta \leq \hat{\theta}, \\ \tan \left(\left|\widetilde{I}_{1}\right|-\varepsilon-\theta\right) & \text { if } \hat{\theta} \leq \theta \leq\left|\widetilde{I}_{1}\right|+\left|\widetilde{J}_{1}\right| / 2-\varepsilon / 2, \\ \tan \left(\theta-\left|\widetilde{I}_{1}\right|-\left|\widetilde{J}_{1}\right|\right) & \text { if }\left|\widetilde{I}_{1}\right|+\left|\widetilde{J}_{1}\right| / 2-\varepsilon / 2 \leq \theta \leq\left|\widetilde{I}_{1}\right|+\left|\widetilde{J}_{1}\right| / 2, \\ \tilde{c}(\theta) & \text { if }\left|\widetilde{I}_{1}\right|+\left|\widetilde{J}_{1}\right| / 2 \leq \theta \leq \pi\end{cases}
$$

We claim that $c^{*}$ belongs to $\mathcal{Z}$ if $\varepsilon$ is small enough. Indeed, $c^{*} \geq 0$ in $I_{1}^{*}=\left[0,\left|\tilde{I}_{1}\right|-\varepsilon\right]$ and $\left|I_{1}^{*}\right| \geq \pi / 3$ as soon as $\varepsilon \leq\left|\widetilde{I}_{1}\right|-\pi / 3$, and moreover the $L^{\infty}$ bound in $I_{1}^{*}$ holds since

$$
\left\|c^{*}\right\|_{L^{\infty}\left(I_{1}^{*}\right)} \leq\|\tilde{c}\|_{L^{\infty}\left(\tilde{I}_{1}\right)} \leq \tan \left(\pi / 6-\tilde{\ell}_{1} / 4\right)<\tan \left(\pi / 6-\ell_{1}^{*} / 4\right) .
$$

It is easily checked that

$$
P\left(c^{*}\right)-P(\tilde{c})=\varepsilon\left(\frac{1}{\cos \left(\left|\widetilde{J}_{1}\right| / 2\right)}-\frac{1}{\cos \left(\left|\widetilde{I}_{1}\right|-\theta^{-}\right)}\right)+o(\varepsilon),
$$

hence by the maximality of $\tilde{c}$ the equality (4.27) follows. 
In a perfectly analogous way, one can prove the same property in a right neighborhood of the left endpoint and in a (possibly empty, in case $\widetilde{J}_{2}=\emptyset$ ) left neighborhood of the right endpoint of $\widetilde{I}_{2}$, that is,

$$
\begin{array}{ll}
\tilde{c}(\theta)=\tan \left(\theta-\left(\left|\widetilde{I}_{1}\right|+\left|\widetilde{J}_{1}\right|\right)\right) & \text { in }\left[\left|\widetilde{I}_{1}\right|+\left|\widetilde{J}_{1}\right|,\left|\widetilde{I}_{1}\right|+3\left|\widetilde{J}_{1}\right| / 2\right], \\
\tilde{c}(\theta)=\tan \left(\left|\widetilde{I}_{1}\right|+\left|\widetilde{J}_{1}\right|+\left|\widetilde{I}_{2}\right|-\theta\right) & \text { in }\left[\left|\widetilde{I}_{1}\right|+\left|\widetilde{J}_{1}\right|+\left|\widetilde{I}_{2}\right|-\left|\widetilde{J}_{2}\right| / 2,\left|\widetilde{I}_{1}\right|+\left|\widetilde{J}_{1}\right|+\left|\widetilde{I}_{2}\right|\right] .
\end{array}
$$

Part IV.3. Conclusion.

Let us consider the function $c^{*}$ drawn in Figure 15, where $\left(c^{*}\right)^{\prime}= \pm\left(1+\left(c^{*}\right)^{2}\right)$ in $\left\{c^{*} \neq 0\right\}$, so that $c^{*}$ is built by suitable translations of $\pm \tan \theta$, and the constants $a, \ldots, f$ are given by

$$
\begin{gathered}
a=\left|\widetilde{I}_{1}\right|-\left|\widetilde{J}_{1}\right|, \quad b=\left|\widetilde{I}_{1}\right|, \quad c=\left|\widetilde{I}_{1}\right|+\left|\widetilde{J}_{1}\right|, \quad d=\left|\widetilde{I}_{1}\right|+2\left|\widetilde{J}_{1}\right|, \\
e=\pi-2\left|\widetilde{J}_{2}\right|, \quad f=\pi-\left|\widetilde{J}_{2}\right| .
\end{gathered}
$$

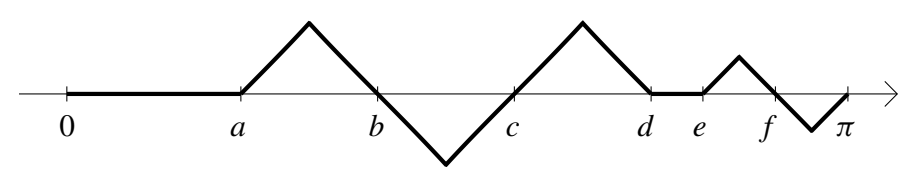

Fig. 15. The function $c^{*}$ in Part IV.3.

Notice that, as in the figure, $e>d$ : indeed,

$$
e=\pi-2\left|\widetilde{J}_{2}\right|=\left|\widetilde{I}_{1}\right|+\left|\widetilde{J}_{1}\right|+\left|\widetilde{I}_{2}\right|-\left|\widetilde{J}_{2}\right|=d+\left|\widetilde{I}_{2}\right|-\left(\left|\widetilde{J}_{1}\right|+\left|\widetilde{J}_{2}\right|\right)>d .
$$

Putting together Parts IV.1 and IV.2, we know that $c^{*} \leq \tilde{c}$ pointwise in $[0, \pi]$, so that

$$
\int_{0}^{\pi} c^{*}(\theta) \sin \theta d \theta \leq \int_{0}^{\pi} \tilde{c}(\theta) \sin \theta d \theta \leq 0 .
$$

However, it is clear that

$$
\int_{e}^{\pi} c^{*}(\theta) \sin \theta d \theta \geq 0
$$

while

$$
\begin{aligned}
\int_{a}^{d} c^{*}(\theta) \sin \theta d \theta & =\int_{a}^{b} c^{*}(\theta)\left(\sin \theta-\sin \left(\theta+\left|\widetilde{J}_{1}\right|\right)+\sin \left(\theta+2\left|\widetilde{J}_{1}\right|\right)\right) d \theta \\
& =\int_{a}^{b} c^{*}(\theta) \sin \left(\theta+\left|\widetilde{J}_{1}\right|\right)\left(2 \cos \left(\left|\widetilde{J}_{1}\right|\right)-1\right) d \theta>0
\end{aligned}
$$

since $\left|\widetilde{J}_{1}\right|<\pi / 3$. Putting together (4.31) and (4.32), we get a contradiction to (4.30), thus finally concluding Part IV.

We can now proceed to the proof of Theorem 3 . 
Proof of Theorem 3. Let $\bar{c}$ be a function maximizing the perimeter, and consider the maximal open intervals in $\mathbb{S}^{1}$ where $\bar{c}$ has a constant sign. Since $P(\bar{c}) \geq P(\hat{c})$ by optimality, Propositions 4.5 and 4.6 ensure that all these intervals are shorter than $\pi / 3$. Then the result follows from Proposition 4.4.

Acknowledgments. We would like to thank Bernd Kawohl for pointing out some of the papers in the bibliography to us.

This research was partially supported by the European Research Council 2008 Advanced Grant Analytic Techniques for Geometric and Functional Inequalities, and by the MEC of Spain Government through the 2008 project MTM2008-03541.

\section{References}

[1] Auerbach, H.: Sur un problème de M. Ulam concernant l'équilibre des corps flottants. Studia Math. 7, 121-142 (1938) Zbl 0018.17504

[2] Croft, H. P., Falconer, K. J., Guy, R. K.: Unsolved Problems in Geometry. Springer (1991) Zbl 0748.52001 MR 1107516

[3] Eggleston, H. G.: The maximal length of chords bisecting the area or perimeter length of plane convex sets. J. London Math. Soc. 36, 122-128 (1961) Zbl 0109.14902 MR 0125494

[4] Geppert, H.: Über einige Kennzeichnungen des Kreises. Math. Z. 46, 117-128 (1940) Zbl 0022.26601 MR 0001594

[5] Goodey, P.: Area and perimeter bisectors of planar convex sets. In: Integral Geometry and Convexity, World Sci., 29-35 (2006) Zbl 1124.52003 MR 2240971

[6] Grüne, A.: Geometric dilation and halving distance. Dissertation zur Erlangung des Doktorgrades der Math.-Nat. Fakultät der Rheinischen Friedrich-Wilhelms-Universität Bonn (2006)

[7] Grüne, A., Klein, R., Miori, C., Segura Gomis, S.: Chords halving the area of a planar convex set. Math. Inequal. Appl. 10, 205-216 (2007) Zbl 1113.52023 MR 2286705

[8] Mampel, K. L.: Über Zindlerkurven. J. Reine Angew. Math. 234, 12-44 (1969) Zbl 0169.23502 MR 0244874

[9] Radziszewski, K.: Sur les cordes qui partagent l'aire d'un ovale en 2 parties égales. Ann. Univ. Mariae Curie-Skłodowska Sect. A 8, 89-92 (1954) Zbl 0071.38005 MR 0080940

[10] Salkowski, E.: Eine kennzeichnende Eigenschaft des Kreises. Sitzungsber. Heidelberger Akad. Wiss. Math.-Nat. Kl. 1934, no. 14, 57-62 Zbl 0010.07701

[11] Zindler, K.: Über konvexe Gebilde, II. Teil. Monatsh. Math. Phys. 31, 25-56 (1921) JFM 48.0833.05 